\title{
Landau equation and QCD sum rules for the tetraquark molecular states
}

\author{
Zhi-Gang Wang* \\ Department of Physics, North China Electric Power University, \\ Baoding 071003, People's Republic of China
}

(Received 28 January 2020; accepted 23 March 2020; published 10 April 2020)

\begin{abstract}
The quarks and gluons are confined objects; they cannot be put on the mass shell. It is questionable to apply the Landau equation to study the Feynman diagrams in the QCD sum rules. Furthermore, we carry out the operator product expansion in the deep Euclidean region $p^{2} \rightarrow-\infty$, where the Landau singularities cannot exist. The Landau equation servers as a kinematical equation in the momentum space and is independent on the factorizable and nonfactorizable properties of the Feynman diagrams in the color space. The meson-meson scattering state and tetraquark molecular state both have four valence quarks, which form two color-neutral clusters; we cannot distinguish the contributions based on the two color-neutral clusters in the factorizable Feynman diagrams. Lucha et al. assert that the contributions at the order $\mathcal{O}\left(\alpha_{s}^{k}\right)$ with $k \leq 1$ in the operator product expansion, which are factorizable in the color space, are exactly canceled out by the meson-meson scattering states at the hadron side, and the tetraquark molecular states begin to receive contributions at the order $\mathcal{O}\left(\alpha_{s}^{2}\right)$. Such an assertion is questionable, we refute the assertion in details, and choose an axial-vector current and a tensor current to examine the outcome of the assertion. After a detailed analysis, we observe that the meson-meson scattering states cannot saturate the QCD sum rules, while the tetraquark molecular states can saturate the QCD sum rules. The Landau equation is of no use to study the Feynman diagrams in the QCD sum rules for the tetraquark molecular states; the tetraquark molecular states begin to receive contributions at the order $\mathcal{O}\left(\alpha_{s}^{0} / \alpha_{s}^{1}\right)$ rather than at the order $\mathcal{O}\left(\alpha_{s}^{2}\right)$.
\end{abstract}

DOI: 10.1103/PhysRevD.101.074011

\section{INTRODUCTION}

In 2003, the Belle Collaboration observed a narrow charmoniumlike structure $X(3872)$ in the $\pi^{+} \pi^{-} J / \psi$ invariant mass spectrum in the exclusive $B$ decays [1], which cannot be accommodated in the traditional or normal quark-antiquark model. Thereafter, more than 20 charmoniumlike exotic states were observed by the BABAR, Belle, BESIII, CDF, CMS, D0, LHCb Collaborations [2]; some exotic states are still needed confirmation and their quantum numbers have not been established yet. There have seen several possible interpretations for those $X, Y$, and $Z$ states, such as the tetraquark states, tetraquark (or hadronic) molecular states, dynamically generated resonances, hadroquarkonium, kinematical effects, cusp effects, etc., [3,4].

Among those possible interpretations, the tetraquark states and tetraquark molecular states are outstanding and attract much attention as the exotic $X, Y$, and $Z$ states lie near the thresholds of two charmed mesons. In 2006,

\footnotetext{
*zgwang@aliyun.com
}

Published by the American Physical Society under the terms of the Creative Commons Attribution 4.0 International license. Further distribution of this work must maintain attribution to the author(s) and the published article's title, journal citation, and DOI. Funded by SCOAP ${ }^{3}$.
Matheus et al. assigned $X(3872)$ to be the $J^{P C}=1^{++}$ diquark-antidiquark type tetraquark state and studied its mass with the QCD sum rules [5]. It is the first time to apply the QCD sum rules to study the exotic $X, Y$, and $Z$ states. Thereafter, the QCD sum rules become a powerful theoretical approach in studying the masses and widths of the exotic $X, Y$, and $Z$ states, irrespective of assigning them as the hidden-charm (or hidden-bottom) tetraquark states or tetraquark (or hadronic) molecular states, and have given many successful descriptions of the hadron properties [4-12]. In the QCD sum rules for the tetraquark states and tetraquark molecular states, we choose the diquarkantidiquark type currents or meson-meson type (more precisely, the color-singlet-color-singlet type currents), respectively, and they can be reformed into each other via Fierz rearrangements, for example,

$$
\begin{aligned}
J_{\mu}= & \frac{\varepsilon^{i j k} \varepsilon^{i m n}}{\sqrt{2}}\left\{u_{j}^{T} C \gamma_{5} c_{k} \bar{d}_{m} \gamma_{\mu} C \bar{c}_{n}^{T}-u_{j}^{T} C \gamma_{\mu} c_{k} \bar{d}_{m} \gamma_{5} C \bar{c}_{n}^{T}\right\}, \\
= & \frac{1}{2 \sqrt{2}}\left\{i \bar{c} i \gamma_{5} c \bar{d} \gamma_{\mu} u-i \bar{c} \gamma_{\mu} c \bar{d} i \gamma_{5} u+\bar{c} u \bar{d} \gamma_{\mu} \gamma_{5} c\right. \\
& -\bar{c} \gamma_{\mu} \gamma_{5} u \bar{d} c-i \bar{c} \gamma^{\nu} \gamma_{5} c \bar{d} \sigma_{\mu \nu} u+i \bar{c} \sigma_{\mu \nu} c \bar{d} \gamma^{\nu} \gamma_{5} u \\
& \left.-i \bar{c} \sigma_{\mu \nu} \gamma_{5} u \bar{d} \gamma^{\nu} c+i \bar{c} \gamma^{\nu} u \bar{d} \sigma_{\mu \nu} \gamma_{5} c\right\},
\end{aligned}
$$

where $i, j, k, m, n$ are color indices. 
In the correlation functions for the color-singlet-colorsinglet type currents, Lucha et al. assert that the Feynman diagrams can be divided into or separated into factorizable diagrams and nonfactorizable diagrams in the color space in the operator product expansion, the contributions at the order $\mathcal{O}\left(\alpha_{s}^{k}\right)$ with $k \leq 1$, which are factorizable in the color space, are exactly canceled out by the meson-meson scattering states at the hadron side, the nonfactorizable diagrams, if have a Landau singularity, begin to make contributions to the tetraquark (molecular) states, the tetraquark (molecular) states begin to receive contributions at the order $\mathcal{O}\left(\alpha_{s}^{2}\right)$ [according to the Fierz rearrangements, see Eq. (1)] [13].

About ten years before the work of Lucha et al., Lee and Kochelev studied the two-pion contributions in the QCD sum rules for the scalar meson $f_{0}(600)$ ) (or $f_{0}(500)$ named by the Particle Data Group now [2]) as the tetraquark state and observed that the contributions of the order $\mathcal{O}\left(\alpha_{s}^{k}\right)$ with $k \leq 1$ cannot be canceled out by the two-pion scattering states [14].

In this paper, we will examine the assertion of Lucha et al. in details and use two examples to illustrate that the Landau equation is of no use in the QCD sum rule for the tetraquark molecular states.

The article is arranged as follows: in Sec. II, we discuss the usefulness of the Landau equation in the QCD sum rules for the tetraquark molecular states; in Sec. III, we obtain the QCD sum rules for the meson-meson scattering states and tetraquark molecular states as an example; in Sec. IV, we present the numerical results and discussions; Sec. V is reserved for our conclusion.

\section{IS LANDAU EQUATION USEFUL IN THE QCD SUM RULES FOR THE TETRAQUARK MOLECULAR STATES?}

In the following, we write down the two-point correlation function $\Pi_{\mu \nu}(p)$ in the QCD sum rules as an example,

$$
\Pi_{\mu \nu}(p)=i \int d^{4} x e^{i p \cdot x}\left\langle 0\left|T\left\{J_{\mu}(x) J_{\nu}^{\dagger}(0)\right\}\right| 0\right\rangle,
$$

where

$$
\begin{aligned}
J_{\mu}(x)= & \frac{1}{\sqrt{2}}\left[\bar{u}(x) i \gamma_{5} c(x) \bar{c}(x) \gamma_{\mu} d(x)\right. \\
& \left.+\bar{u}(x) \gamma_{\mu} c(x) \bar{c}(x) i \gamma_{5} d(x)\right] .
\end{aligned}
$$

The color-singlet-color-singlet type current $J_{\mu}(x)$ has the quantum numbers $J^{P C}=1^{+-}$, at the hadron side, the quantum field theory allows nonvanishing couplings to the $D \bar{D}^{*}+D^{*} \bar{D}$ scattering states or tetraquark molecular states with $J^{P C}=1^{+-}$.

At the QCD side, when we carry out the operator product expansion, Lucha et al. assert that the Feynman diagrams can be divided into or separated into factorizable diagrams

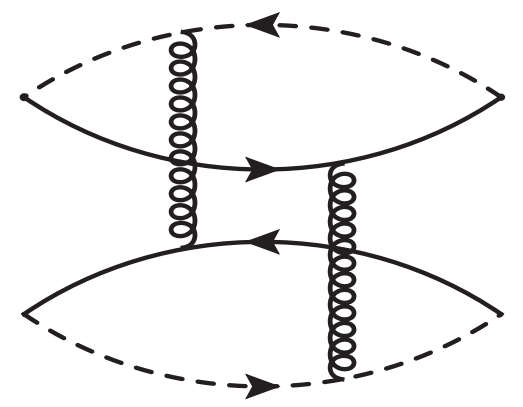

FIG. 1. The nonfactorizable Feynman diagrams of the order $\mathcal{O}\left(\alpha_{s}^{2}\right)$ for the color-singlet-color-singlet type currents, other diagrams obtained by interchanging of the heavy quark lines (dashed lines) and light quark lines (solid lines) are implied.

and nonfactorizable diagrams, the Feynman diagrams of the orders $\mathcal{O}\left(\alpha_{s}^{0}\right)$ and $\mathcal{O}\left(\alpha_{s}^{1}\right)$ are factorizable, the factorizable diagrams are exactly canceled out by the meson-meson scattering states, while the nonfactorizable Feynman diagrams, which are of the order $\mathcal{O}\left(\alpha_{s}^{2}\right)$, if have a Landau singularity, begin to make contributions to the tetraquark (molecular) states, the tetraquark (molecular) states begin to receive contributions at the order $\mathcal{O}\left(\alpha_{s}^{2}\right)$ [13]; see the Feynman diagrams shown in Fig. 1. In fact, such an assertion is questionable.

First, we cannot assert that the factorizable Feynman diagrams in color space are exactly canceled out by the meson-meson scattering states, because the meson-meson scattering state and tetraquark molecular state both have four valence quarks, which can be divided into or separated into two color-neutral clusters. We cannot distinguish which Feynman diagrams contribute to the meson-meson scattering state or tetraquark molecular state based on the two color-neutral clusters.

Second, the quarks and gluons are confined objects, they cannot be put on the mass shell; it is questionable to assert that the Landau equation is applicable in the nonperturbative calculations dealing with the quark-gluon bound states [15].

If we insist on applying the Landau equation to study the Feynman diagrams in the QCD sum rules, we should choose the pole masses rather than the $\overline{\mathrm{MS}}$ masses to warrant that there exists a mass pole which corresponds to the mass shell in pure perturbative calculations, just like in the quantum electrodynamics, where the electron, muon, and tau can be put on the mass shell.

According to the assertion of Lucha et al., the tetraquark (molecular) states begin to receive contributions at the order $\mathcal{O}\left(\alpha_{s}^{2}\right)$ [13]; it is reasonable to take the pole masses $\hat{m}_{Q}$ as

$$
\begin{aligned}
\hat{m}_{Q}= & m_{Q}\left(m_{Q}\right)\left[1+\frac{4}{3} \frac{\alpha_{s}\left(m_{Q}\right)}{\pi}+f\left(\frac{\alpha_{s}\left(m_{Q}\right)}{\pi}\right)^{2}\right. \\
& \left.+g\left(\frac{\alpha_{s}\left(m_{Q}\right)}{\pi}\right)^{3}\right]
\end{aligned}
$$


to put the heavy quark lines on the mass shell; the explicit expressions of the coefficients $f$ and $g$ can be found in Refs. [2,16]. It is straightforward to obtain $\hat{m}_{b}=$ $m_{b}\left(m_{b}\right)(1+0.10+0.05+0.03)=4.78 \pm 0.06 \mathrm{GeV}[2]$.

If the Landau equation is applicable in the QCD sum rules for the tetraquark states and tetraquark molecular states, it is certainly applicable in the QCD sum rules for the traditional or normal charmonium and bottomonium states. In the case of the $c$ quark, the pole mass $\hat{m}_{c}=1.67 \pm$ $0.07 \mathrm{GeV}$ from the Particle Data Group [2], the Landau singularity appears at the $s$ channel $\sqrt{s}=\sqrt{p^{2}}=2 \hat{m}_{c}=$ $3.34 \pm 0.14 \mathrm{GeV}>m_{\eta_{c}}$ and $m_{J / \psi}$. While in the case of the $b$ quark, the pole mass $\hat{m}_{b}=4.78 \pm 0.06 \mathrm{GeV}$ from the Particle Data Group [2], the Landau singularity appears at the $s$ channel $\sqrt{s}=\sqrt{p^{2}}=2 \hat{m}_{b}=9.56 \pm 0.12 \mathrm{GeV}>m_{\eta_{b}}$ and $m_{\Upsilon}$. It is odd or unreliable that the masses of the charmonium (bottomonium) states lie below the threshold $2 \hat{m}_{c}\left(2 \hat{m}_{b}\right)$ in the QCD sum rules for $\eta_{c}$ and $J / \psi\left(\eta_{b}\right.$ and $\Upsilon$ ), as the integrals of the forms

$$
\begin{aligned}
& \int_{4 \hat{m}_{c}^{2}}^{s_{0}} \delta\left(s-m_{\eta_{c} / J / \psi}^{2}\right) \exp \left(-\frac{s}{T^{2}}\right) d s, \\
& \int_{4 \hat{m}_{b}^{2}}^{s_{0}} \delta\left(s-m_{\eta_{b} / \Upsilon}^{2}\right) \exp \left(-\frac{s}{T^{2}}\right) d s
\end{aligned}
$$

at the hadron side are meaningless, where $T^{2}$ is the Borel parameter. The tiny widths of the $\eta_{c}, J / \psi, \eta_{b}$, and $\Upsilon$ valuate the zero-width approximation; the hadronic spectral densities are of the form $\delta\left(s-m_{\left.\eta_{c} / J / \psi / \eta_{b} / \Upsilon\right)}^{2}\right.$.

Third, the nonfactorizable Feynman diagrams which have the Landau singularities begin to appear at the order $\mathcal{O}\left(\alpha_{s}^{0} / \alpha_{s}^{1}\right)$ rather than at the order $\mathcal{O}\left(\alpha_{s}^{2}\right)$ and make contributions to the tetraquark molecular states, if the assertion (the nonfactorizable Feynman diagrams which have Landau singularities make contributions to the tetraquark molecular states) of Lucha et al. is right.

The nonperturbative contributions play an important role and serve as a hallmark for the nonperturbative nature of the QCD sum rules; the nonfactorizable contributions appear at the order $\mathcal{O}\left(\alpha_{s}\right)$ due to the operators $\bar{q} g_{s} G q \bar{q} g_{s} G q$, which come from the Feynman diagrams shown in Fig. 2. Such Feynman diagrams can be taken as annihilation diagrams, which play an important role in the tetraquark molecular states [17]. If we insist on applying the Landau equation to study the Feynman diagrams shown in Fig. 2 and choose the pole mass of the $c$ quark, we obtain a subleading Landau singularity at the $s$ channel $s=p^{2}=\left(\hat{m}_{c}+\hat{m}_{c}\right)^{2}$, which indicates that it contributes to the tetraquark molecular states. From the operators $\bar{q} g_{s} G q \bar{q} g_{s} G q$, we can obtain the vacuum condensate $\left\langle\bar{q} g_{s} \sigma G q\right\rangle^{2}$, where $g_{s}^{2}=4 \pi \alpha_{s}$ is absorbed into the vacuum condensate, so the Feynman diagrams in Fig. 2 can be counted as of the order $\mathcal{O}\left(\alpha_{s}^{0}\right)$. The nonfactorizable Feynman diagrams appear at the order $\mathcal{O}\left(\alpha_{s}^{0}\right)$ or $\mathcal{O}\left(\alpha_{s}^{1}\right)$ (based on how to account for the $g_{s}^{2}$ in the

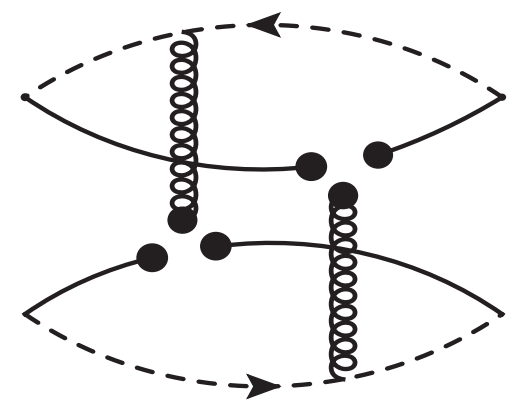

FIG. 2. The nonfactorizable Feynman diagrams contribute to the vacuum condensates $\left\langle\bar{q} g_{s} \sigma G q\right\rangle^{2}$ for the color-singlet-colorsinglet type currents, where the solid lines and dashed lines denote the light quarks and heavy quarks, respectively.

vacuum condensates), not at the order $\mathcal{O}\left(\alpha_{s}^{2}\right)$ asserted in Ref. [13].

Fourth, the Landau equation servers as a kinematical equation in the momentum space and is independent on the factorizable and nonfactorizable properties of the Feynman diagrams in the color space. Without taking it for granted that the factorizable Feynman diagrams in the color space only make contributions to the two-meson scattering states, the Landau equation cannot exclude the factorizable Feynman diagrams in the color space; those diagrams can also have the Landau singularities.

In the leading order, the factorizable Feynman diagrams shown in Fig. 3 can be divided into or separated into two color-neutral clusters; each cluster corresponds to a trace both in the color space and in the Dirac spinor space. However, in the momentum space, they are nonfactorizable diagrams; the basic integrals are of the form

$$
\begin{aligned}
& \int d^{4} q d^{4} k d^{4} l \frac{1}{(p+q-k+l)^{2}-m_{c}^{2}} \frac{1}{q^{2}-m_{q}^{2}} \frac{1}{k^{2}-m_{q}^{2}} \\
& \times \frac{1}{l^{2}-m_{c}^{2}} .
\end{aligned}
$$

If we choose the pole masses, there exists a Landau singularity or an $s$ channel singularity at $s=p^{2}=$ $\left(\hat{m}_{u}+\hat{m}_{d}+\hat{m}_{c}+\hat{m}_{c}\right)^{2}$, which is just a signal of a fourquark intermediate state. We cannot assert that it is a signal of a meson-meson scattering state or a tetraquark molecular state, because the meson-meson scattering state and tetraquark molecular state both have four valence quarks, $q, \bar{q}$, $c$, and $\bar{c}$, which form two color-neutral clusters. The Landau singularity is just a kinematical singularity, not a dynamical singularity [18]; it is useless in distinguishing the contributions to the meson-meson scattering state and tetraquark molecular state. If we switch off the assertion that the factorizable Feynman diagrams shown in Fig. 3 make contributions to the meson-meson scattering states alone, the $s$-channel singularity at $s=p^{2}=\left(\hat{m}_{u}+\hat{m}_{d}+\right.$ $\left.\hat{m}_{c}+\hat{m}_{c}\right)^{2}$ supports that they also contribute to the tetraquark molecular states. 


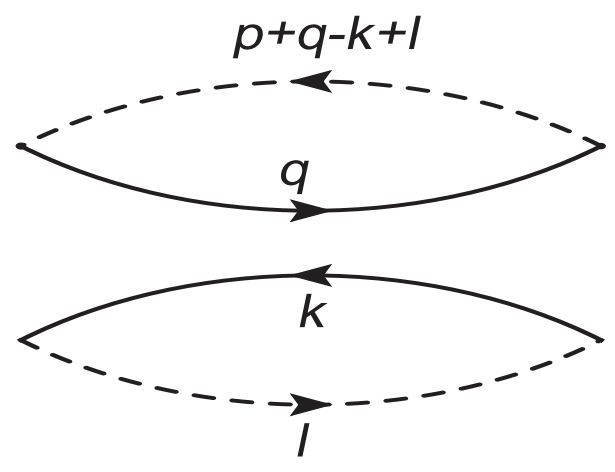

FIG. 3. The Feynman diagrams for the lowest order contributions, where the solid lines and dashed lines represent the light quarks and heavy quarks, respectively.

Fifth, only formal QCD sum rules for the tetraquark states or tetraquark molecular states are obtained based on the assertion of Lucha et al. in Ref. [13]; no feasible QCD sum rules with predictions can be confronted to the experimental data obtained up to now.

Sixth, in the QCD sum rules, we carry out the operator product expansion in the deep Euclidean space, $-p^{2} \rightarrow \infty$, then obtain the physical spectral densities at the quarkgluon level through dispersion relation [19-21],

$$
\rho_{\mathrm{QCD}}(s)=\left.\frac{1}{\pi} \operatorname{Im} \Pi(s+i \epsilon)\right|_{\epsilon \rightarrow 0},
$$

where $\Pi(s)$ denotes the correlation functions. The Landau singularities require that the squared momentum $p^{2}=$ $\left(\hat{m}_{u}+\hat{m}_{d}+\hat{m}_{c}+\hat{m}_{c}\right)^{2}$ in the Feynman diagrams; see Fig. 3 and Eq. (6). It is questionable to perform the operator product expansion.

\section{QCD SUM RULES WITH COLOR- SINGLET-COLOR-SINGLET TYPE CURRENTS}

Now, let us assume that the assertion of Lucha et al. is right; the tetraquark molecular states begin to receive contributions at the order $\mathcal{O}\left(\alpha_{s}^{2}\right)$; the contributions at the order $\mathcal{O}\left(\alpha_{s}^{k}\right)$ with $k \leq 1$ are exactly canceled out by the meson-meson scattering states. We saturate the QCD sum rules with the meson-meson scattering states and examine whether or not we can obtain feasible QCD sum rules.

In the following, we write down the two-point correlation functions $\Pi_{\mu \nu}(p)$ and $\Pi_{\mu \nu \alpha \beta}(p)$ in the QCD sum rules,

$$
\begin{gathered}
\Pi_{\mu \nu}(p)=i \int d^{4} x e^{i p \cdot x}\left\langle 0\left|T\left\{J_{\mu}(x) J_{\nu}^{\dagger}(0)\right\}\right| 0\right\rangle, \\
\Pi_{\mu \nu \alpha \beta}(p)=i \int d^{4} x e^{i p \cdot x}\left\langle 0\left|T\left\{J_{\mu \nu}(x) J_{\alpha \beta}^{\dagger}(0)\right\}\right| 0\right\rangle,
\end{gathered}
$$

where

$$
\begin{aligned}
J_{\mu}(x)= & \frac{1}{\sqrt{2}}\left[\bar{u}(x) i \gamma_{5} c(x) \bar{c}(x) \gamma_{\mu} d(x)\right. \\
& \left.+\bar{u}(x) \gamma_{\mu} c(x) \bar{c}(x) i \gamma_{5} d(x)\right], \\
J_{\mu \nu}(x)= & \frac{1}{\sqrt{2}}\left[\bar{s}(x) \gamma_{\mu} c(x) \bar{c}(x) \gamma_{\nu} \gamma_{5} s(x)\right. \\
& \left.-\bar{s}(x) \gamma_{\nu} \gamma_{5} c(x) \bar{c}(x) \gamma_{\mu} s(x)\right] .
\end{aligned}
$$

The current $J_{\mu}(x)$ has the quantum numbers $J^{P C}=1^{+-}$, while the current $J_{\mu \nu}(x)$ has definite charge conjugation, the components $J_{0 i}(x)$ and $J_{i j}(x)$ have positive parity and negative parity, respectively, where the space indexes $i$, $j=1,2,3$. The charged current $J_{\mu}(x)$ couples potentially to the $D \bar{D}^{*}+D^{*} \bar{D}$ scattering state or tetraquark molecular state with $J^{P C}=1^{+-}$, while the neutral current $J_{\mu \nu}(x)$ couples potentially to the $D_{s}^{*} \bar{D}_{s 1}-D_{s 1} \bar{D}_{s}^{*}$ meson-meson scattering states or tetraquark molecular states with $J^{P C}=$ $1^{++}$and $1^{-+}$. Thereafter, we will denote the charged $D \bar{D}^{*}+D^{*} \bar{D}$ tetraquark molecular state with $J^{P C}=1^{+-}$ as $Z_{c}$ and denote the neutral $D_{s}^{*} \bar{D}_{s 1}-D_{s 1} \bar{D}_{s}^{*}$ tetraquark molecular states with $J^{P C}=1^{++}$and $1^{-+}$as $X_{c}^{+}$and $X_{c}^{-}$, respectively, where the superscripts \pm on the $X_{c}^{ \pm}$denote the positive parity and negative parity, respectively.

In the following, we write down the possible currenthadron couplings explicitly:

$$
\begin{aligned}
\left\langle 0\left|J_{\mu}(0)\right| D(q) \bar{D}^{*}(p-q)\right\rangle & =\frac{1}{\sqrt{2}} \frac{f_{D} m_{D}^{2}}{m_{c}} f_{D^{*}} m_{D^{*}} \varepsilon_{\mu}(p-q), \\
\left\langle 0\left|J_{\mu}(0)\right| D^{*}(q) \bar{D}(p-q)\right\rangle & =\frac{1}{\sqrt{2}} \frac{f_{D} m_{D}^{2}}{m_{c}} f_{D^{*}} m_{D^{*}} \varepsilon_{\mu}(q), \\
\left\langle 0\left|J_{\mu}(0)\right| D(q) \bar{D}_{0}(p-q)\right\rangle & =\frac{1}{\sqrt{2}} \frac{f_{D} m_{D}^{2}}{m_{c}} f_{D_{0}}(p-q)_{\mu}, \\
\left\langle 0\left|J_{\mu}(0)\right| D_{0}(q) \bar{D}(p-q)\right\rangle & =\frac{1}{\sqrt{2}} \frac{f_{D} m_{D}^{2}}{m_{c}} f_{D_{0}} q_{\mu}, \\
\left\langle 0\left|J_{\mu}(0)\right| Z_{c}(p)\right\rangle & =\lambda_{Z} \varepsilon_{\mu}(p),
\end{aligned}
$$

$$
\begin{aligned}
\left\langle 0\left|J_{\mu \nu}(0)\right| D_{s}^{*}(q) \bar{D}_{s 1}(p-q)\right\rangle & =\frac{1}{\sqrt{2}} f_{D_{s}^{*}} m_{D_{s}^{*}} f_{D_{s 1}} m_{D_{s_{1}}} \varepsilon_{\mu}(q) \varepsilon_{\nu}(p-q), \\
\left\langle 0\left|J_{\mu \nu}(0)\right| D_{s 1}(q) \bar{D}_{s}^{*}(p-q)\right\rangle & =-\frac{1}{\sqrt{2}} f_{D_{s 1}} m_{D_{s_{1}}} f_{D_{s}^{*}} m_{D_{s}^{*}} \varepsilon_{\nu}(q) \varepsilon_{\mu}(p-q), \\
\left\langle 0\left|J_{\mu \nu}(0)\right| D_{s}^{*}(q) \bar{D}_{s}(p-q)\right\rangle & =\frac{i}{\sqrt{2}} f_{D_{s}^{*}} m_{D_{s}^{*}} f_{D_{s}} \varepsilon_{\mu}(q)(p-q)_{\nu},
\end{aligned}
$$




$$
\begin{aligned}
\left\langle 0\left|J_{\mu \nu}(0)\right| D_{s}(q) \bar{D}_{s}^{*}(p-q)\right\rangle & =-\frac{i}{\sqrt{2}} f_{D_{s}} f_{D_{s}^{*}} m_{D_{s}^{*}} q_{\nu} \varepsilon_{\mu}(p-q), \\
\left\langle 0\left|J_{\mu \nu}(0)\right| D_{s 0}(q) \bar{D}_{s}(p-q)\right\rangle & =\frac{i}{\sqrt{2}} f_{D_{s 0}} f_{D_{s}} q_{\mu}(p-q)_{\nu}, \\
\left\langle 0\left|J_{\mu \nu}(0)\right| D_{s}(q) \bar{D}_{s 0}(p-q)\right\rangle & =-\frac{i}{\sqrt{2}} f_{D_{s}} f_{D_{s 0}} q_{\nu}(p-q)_{\mu},
\end{aligned}
$$

$$
\begin{aligned}
\left\langle 0\left|J_{\mu \nu}(0)\right| X_{c}^{-}(p)\right\rangle & =\frac{\lambda_{X^{-}}}{M_{X^{-}}} \varepsilon_{\mu \nu \alpha \beta} \varepsilon^{\alpha}(p) p^{\beta}, \\
\left\langle 0\left|J_{\mu \nu}(0)\right| X_{c}^{+}(p)\right\rangle & =\frac{\lambda_{X^{+}}}{M_{X^{+}}}\left[\varepsilon_{\mu}(p) p_{\nu}-\varepsilon_{\nu}(p) p_{\mu}\right],
\end{aligned}
$$

$\varepsilon_{\mu}$ are the polarization vectors of the vector and axial-vector mesons or tetraquark molecular states, $f_{D}, f_{D_{s}}, f_{D^{*}}, f_{D_{s}^{*}}$, $f_{D_{0}}, f_{D_{s 0}}$, and $f_{D_{s 1}}$ are the decay constants of the traditional or normal heavy mesons, and $\lambda_{Z}$ and $\lambda_{X^{ \pm}}$are the pole residues of the tetraquark molecular states. The charged $D \bar{D}^{*}+D^{*} \bar{D}$ tetraquark molecular state $Z_{c}$ with $J^{P C}=1^{+-}$and the neutral $D_{s}^{*} \bar{D}_{s 1}-D_{s 1} \bar{D}_{s}^{*}$ tetraquark molecular state $X_{c}^{-}$with $J^{P C}=$ $1^{-+}$differ from the traditional mesons significantly and are good subjects to study the exotic states.

Now, we take a short digression to give some explanations for the definitions of the current-hadron couplings in Eqs. (12) and (14). First, let us write down the standard definitions for the decay constants of the traditional or normal heavy mesons,

$$
\begin{aligned}
\left\langle 0\left|\bar{q}(0) i \gamma_{5} c(0)\right| D(q)\right\rangle & =\frac{f_{D} m_{D}^{2}}{m_{c}}, \\
\left\langle 0\left|\bar{q}(0) \gamma_{\mu} c(0)\right| D^{*}(q)\right\rangle & =f_{D^{*}} m_{D^{*}} \varepsilon_{\mu}(q), \\
\left\langle 0\left|\bar{s}(0) \gamma_{\mu} c(0)\right| D_{s}^{*}(q)\right\rangle & =f_{D_{s}^{*}} m_{D_{s}^{*}} \varepsilon_{\mu}(q), \\
\left\langle 0\left|\bar{q}(0) \gamma_{\mu} c(0)\right| D_{0}(q)\right\rangle & =f_{D_{0}} q_{\mu}, \\
\left\langle 0\left|\bar{s}(0) \gamma_{\mu} c(0)\right| D_{s 0}(q)\right\rangle & =f_{D_{s 0}} q_{\mu}, \\
\left\langle 0\left|\bar{s}(0) \gamma_{\mu} \gamma_{5} c(0)\right| D_{s 1}(q)\right\rangle & =f_{D_{s 1}} m_{D_{s 1}} \varepsilon_{\mu}(q), \\
\left\langle 0\left|\bar{s}(0) \gamma_{\mu} \gamma_{5} c(0)\right| D_{s}(q)\right\rangle & =i f_{D_{s}} q_{\mu},
\end{aligned}
$$

based on the properties of the vector currents and axialvector currents and their conservation features, where $q=u, d$. On the other hand, the heavy meson fields have the properties,

$$
\begin{aligned}
\left\langle 0\left|D_{(s / 0 / s 0)}(0)\right| D_{(s / 0 / s 0)}(q)\right\rangle & =1, \\
\left\langle 0\left|D_{(s) \mu}^{*}(0)\right| D_{(s)}^{*}(q)\right\rangle & =\varepsilon_{\mu}(q), \\
\left\langle 0\left|D_{s 1, \mu}(0)\right| D_{s 1}(q)\right\rangle & =\varepsilon_{\mu}(q),
\end{aligned}
$$

which imply that $\bar{q}(x) i \gamma_{5} c(x)=\frac{f_{D} m_{D}^{2}}{m_{c}} D(x)+\cdots$, etc., at the hadron degrees of freedom. From Eqs. (13) and (15)(17), we can express the four-quark currents $J_{\mu}(x)$ and $J_{\mu \nu}(x)$ in terms of the heavy meson fields [in other words, Eq. (13) and (15)-(17) imply that],

$$
\begin{aligned}
J_{\mu}(x)= & \frac{1}{\sqrt{2}} \frac{f_{D} m_{D}^{2}}{m_{c}} f_{D^{*}} m_{D^{*}}\left[D^{0}(x) D_{\mu}^{*-}(x)+D_{\mu}^{* 0}(x) D^{-}(x)\right] \\
& +\frac{1}{\sqrt{2}} \frac{f_{D} m_{D}^{2}}{m_{c}} f_{D_{0}}\left[D^{0}(x) i \partial_{\mu} D_{0}^{-}(x)\right. \\
& \left.+i \partial_{\mu} D_{0}^{0}(x) D^{-}(x)\right]+\lambda_{Z} Z_{c, \mu}(x)+\cdots,
\end{aligned}
$$

$$
\begin{aligned}
J_{\mu \nu}(x)= & \frac{1}{\sqrt{2}} f_{D_{s}^{*}} m_{D_{s}^{*}} f_{D_{s 1}} m_{D_{s 1}}\left[D_{s, \mu}^{*+}(x) D_{s 1, \nu}^{-}(x)-D_{s 1, \nu}^{+}(x) D_{s, \mu}^{*-}(x)\right] \\
& -\frac{1}{\sqrt{2}} f_{D_{s}^{*}} m_{D_{s}^{*}} f_{D_{s}}\left[D_{s, \mu}^{*+}(x) \partial_{\nu} D_{s}^{-}(x)-\partial_{\nu} D_{s}^{+}(x) D_{s, \mu}^{*-}(x)\right] \\
& +\frac{1}{\sqrt{2}} f_{D_{s 0}} f_{D_{s 1}} m_{D_{s 1}}\left[i \partial_{\mu} D_{s 0}^{+}(x) D_{s 1, \nu}^{-}(x)-D_{s 1, \nu}^{+}(x) i \partial_{\mu} D_{s 0}^{-}(x)\right] \\
& -\frac{1}{\sqrt{2}} f_{D_{s 0}} f_{D_{s}}\left[i \partial_{\mu} D_{s 0}^{+}(x) \partial_{\nu} D_{s}^{-}(x)-\partial_{\nu} D_{s}^{+}(x) i \partial_{\mu} D_{s 0}^{-}(x)\right] \\
& -\frac{\lambda_{X^{-}}}{M_{X^{-}}} \varepsilon_{\mu \nu \alpha \beta} i \partial^{\alpha} X_{c}^{-\beta}(x)-\frac{\lambda_{X^{+}}}{M_{X^{+}}}\left[i \partial_{\mu} X_{c, \nu}^{+}(x)-i \partial_{\nu} X_{c, \mu}^{+}(x)\right]+\cdots,
\end{aligned}
$$


according to the assumption of current-hadron duality. It is straightforward to obtain the current-hadron couplings in Eqs. (12) and (14).

At the hadron side, we insert a complete set of intermediate hadronic states with the same quantum numbers as the current operators $J_{\mu}(x)$ and $J_{\mu \nu}(x)$ into the correlation functions $\Pi_{\mu \nu}(p)$ and $\Pi_{\mu \nu \alpha \beta}(p)$ to obtain the hadronic representation $[19,20]$. We isolate the contributions of the meson-meson scattering states and the lowest axialvector and vector tetraquark states according to Eqs. (12)(15), and get the results,

$$
\begin{gathered}
\Pi_{\mu \nu}(p)=\Pi\left(p^{2}\right)\left(-g_{\mu \nu}+\frac{p_{\mu} p_{\nu}}{p^{2}}\right)+\cdots, \\
\Pi_{\mu \nu \alpha \beta}(p)=\Pi_{-}\left(p^{2}\right)\left(g_{\mu \alpha} g_{\nu \beta}-g_{\mu \beta} g_{\nu \alpha}-g_{\mu \alpha} \frac{p_{\nu} p_{\beta}}{p^{2}}-g_{\nu \beta} \frac{p_{\mu} p_{\alpha}}{p^{2}}+g_{\mu \beta} \frac{p_{\nu} p_{\alpha}}{p^{2}}+g_{\nu \alpha} \frac{p_{\mu} p_{\beta}}{p^{2}}\right) \\
+\Pi_{+}\left(p^{2}\right)\left(-g_{\mu \alpha} \frac{p_{\nu} p_{\beta}}{p^{2}}-g_{\nu \beta} \frac{p_{\mu} p_{\alpha}}{p^{2}}+g_{\mu \beta} \frac{p_{\nu} p_{\alpha}}{p^{2}}+g_{\nu \alpha} \frac{p_{\mu} p_{\beta}}{p^{2}}\right),
\end{gathered}
$$

where

$$
\begin{aligned}
\left.\Pi_{(} p^{2}\right) & =\frac{\lambda_{Z}^{2}}{M_{Z}^{2}-p^{2}}+\Pi_{T W}\left(p^{2}\right)+\cdots, \\
\Pi_{-}\left(p^{2}\right) & =P_{-}^{\mu \nu \alpha \beta} \Pi_{\mu \nu \alpha \beta}(p)=\frac{\lambda_{X^{-}}^{2}}{M_{X^{-}}^{2}-p^{2}}+\Pi_{T W}^{-}\left(p^{2}\right)+\cdots, \\
\Pi_{+}\left(p^{2}\right) & =P_{+}^{\mu \nu \alpha \beta} \Pi_{\mu \nu \alpha \beta}(p)=\frac{\lambda_{X^{+}}^{2}}{M_{X^{+}}^{2}-p^{2}}+\cdots,
\end{aligned}
$$

we project out the components $\Pi_{-}\left(p^{2}\right)$ and $\Pi_{+}\left(p^{2}\right)$ by introducing the operators $P_{-}^{\mu \nu \alpha \beta}$ and $P_{+}^{\mu \nu \alpha \beta}$, respectively,

$$
\begin{aligned}
& P_{-}^{\mu \nu \alpha \beta}=\frac{1}{6}\left(g^{\mu \alpha}-\frac{p^{\mu} p^{\alpha}}{p^{2}}\right)\left(g^{\nu \beta}-\frac{p^{\nu} p^{\beta}}{p^{2}}\right), \\
& P_{+}^{\mu \nu \alpha \beta}=\frac{1}{6}\left(g^{\mu \alpha}-\frac{p^{\mu} p^{\alpha}}{p^{2}}\right)\left(g^{\nu \beta}-\frac{p^{\nu} p^{\beta}}{p^{2}}\right)-\frac{1}{6} g^{\mu \alpha} g^{\nu \beta}, \\
& \Pi_{T W}\left(p^{2}\right)=\frac{\lambda_{D D^{*}}^{2}}{16 \pi^{2}} \int_{\Delta_{1}^{2}}^{s_{0}} d s \frac{1}{s-p^{2}} \frac{\sqrt{\lambda\left(s, m_{D}^{2}, m_{D^{*}}^{2}\right)}}{s}\left[1+\frac{\lambda\left(s, m_{D}^{2}, m_{D^{*}}^{2}\right)}{12 s m_{D^{*}}^{2}}\right] \\
& +\frac{\lambda_{D D_{0}}^{2}}{16 \pi^{2}} \int_{\Delta_{2}^{2}}^{s_{0}} d s \frac{1}{s-p^{2}} \frac{\sqrt{\lambda\left(s, m_{D}^{2}, m_{D_{0}}^{2}\right)}}{s} \frac{\lambda\left(s, m_{D}^{2}, m_{D_{0}}^{2}\right)}{12 s}+\cdots, \\
& \Pi_{T W}^{-}\left(p^{2}\right)=\frac{\lambda_{D_{s}^{*} D_{s 1}}^{2}}{16 \pi^{2}} \int_{\Delta_{3}^{2}}^{s_{0}} d s \frac{1}{s-p^{2}} \frac{\sqrt{\lambda\left(s, m_{D_{s}^{*}}^{2}, m_{D_{s 1}}^{2}\right)}}{s}\left[1+\frac{\lambda\left(s, m_{D_{s}^{*}}^{2}, m_{D_{s 1}}^{2}\right.}{12 s m_{D_{s}^{*}}^{2}} \frac{\lambda\left(s, m_{D_{s}^{*}}^{2}, m_{D_{s 1}}^{2}\right)}{12 s m_{D_{s 1}}^{2}}\right] \\
& +\frac{\lambda_{D_{s}^{*} D_{s}}^{2}}{16 \pi^{2}} \int_{\Delta_{4}^{2}}^{s_{0}} d s \frac{1}{s-p^{2}} \frac{\sqrt{\lambda\left(s, m_{D_{s}^{*}}^{2}, m_{D_{s}}^{2}\right)}}{s} \frac{\lambda\left(s, m_{D_{s}^{*}}^{2}, m_{D_{s}}^{2}\right)}{12 s} \\
& +\frac{\lambda_{D_{s 0} D_{s 1}}^{2}}{16 \pi^{2}} \int_{\Delta_{5}^{2}}^{s_{0}} d s \frac{1}{s-p^{2}} \frac{\left.\sqrt{\lambda\left(s, m_{D_{s 0}}^{2}, m_{D_{s 1}}^{2}\right.}\right)}{s} \frac{\lambda\left(s, m_{D_{s 0}}^{2}, m_{D_{s 1}}^{2}\right)}{12 s}+\cdots,
\end{aligned}
$$




$$
\begin{aligned}
\lambda_{D D^{*}}^{2} & =\frac{f_{D}^{2} m_{D}^{4} f_{D^{*}}^{2} m_{D^{*}}^{2},}{m_{c}^{2}}, \\
\lambda_{D D_{0}}^{2} & =\frac{f_{D}^{2} m_{D}^{4} f_{D_{0}}^{2}}{m_{c}^{2}}, \\
\lambda_{D_{s}^{*} D_{s 1}}^{2} & =f_{D_{s}^{*}}^{2} m_{D_{s}^{*}}^{2} f_{D_{s 1}}^{2} m_{D_{s 1}}^{2}, \\
\lambda_{D_{s}^{*} D_{s}}^{2} & =f_{D_{s}^{*}}^{2} m_{D_{s}^{*}}^{2} f_{D_{s}}^{2}, \\
\lambda_{D_{s 0} D_{s 1}}^{2} & =f_{D_{s 0}}^{2} f_{D_{s 1}}^{2} m_{D_{s 1}}^{2}, \\
\Delta_{1}^{2} & =\left(m_{D}+m_{D^{*}}\right)^{2}, \\
\Delta_{2}^{2} & =\left(m_{D}+m_{D_{0}}\right)^{2}, \\
\Delta_{3}^{2} & =\left(m_{D_{s}^{*}}+m_{D_{s 1}}\right)^{2}, \\
\Delta_{4}^{2} & =\left(m_{D_{s}^{*}}+m_{D_{s}}\right)^{2}, \\
\Delta_{5}^{2} & =\left(m_{D_{s 0}}+m_{D_{s 1}}\right)^{2},
\end{aligned}
$$

$\lambda(a, b, c)=a^{2}+b^{2}+c^{2}-2 a b-2 b c-2 c a$. The components $\Pi_{-}\left(p^{2}\right)$ and $\Pi_{+}\left(p^{2}\right)$ receive contributions from the $D_{s}^{*} \bar{D}_{s 1}-D_{s 1} \bar{D}_{s}^{*}$ meson-meson scattering states or tetraquark molecular states with $J^{P C}=1^{-+}$and $1^{++}$, respectively. The conventional hidden-flavor mesons have the normal quantum numbers, $J^{P C}=0^{-+}, 0^{++}, 1^{--}, 1^{+-}$, $1^{++}, 2^{--}, 2^{-+}, 2^{++}, \cdots$. The component $\Pi_{-}\left(p^{2}\right)$ receives contributions with the exotic quantum numbers $J^{P C}=1^{-+}$, while the component $\Pi_{+}\left(p^{2}\right)$ receives contributions with the normal quantum numbers $J^{P C}=1^{++}$. In this paper, we study the tetraquark molecular states (in other words, the exotic states), it is better to choose the component $\Pi_{-}\left(p^{2}\right)$ with the exotic quantum numbers $J^{P C}=1^{-+}$, so we discard the component $\Pi_{+}\left(p^{2}\right)$ with the normal quantum numbers $J^{P C}=1^{++}$. Thereafter, we will neglect the superscript - in the $X_{c}^{-}$for simplicity.

Now, we give some explanations for the components $\Pi_{T W}\left(p^{2}\right)$ and $\Pi_{T W}^{-}\left(p^{2}\right)$ in Eqs. (24) and (25). We draw up the Feynman diagrams for the two-meson scattering state contributions in the correlation functions $\Pi_{\mu \nu}(p)$ and $\Pi_{\mu \nu \alpha \beta}(p)$, see Fig. 4, and resort to the Cutkosky's rule to calculate the imaginary parts $\operatorname{Im} \Pi_{T W}\left(p^{2}\right)$ and $\operatorname{Im} \Pi_{T W}^{-}\left(p^{2}\right)$ with the simple replacements of the two heavy-meson lines,

$$
\begin{aligned}
\frac{1}{q^{2}-m_{A}^{2}+i \epsilon} & \rightarrow-2 \pi i \delta\left(q^{2}-m_{A}^{2}\right), \\
\frac{1}{(p-q)^{2}-m_{B}^{2}+i \epsilon} & \rightarrow-2 \pi i \delta\left((p-q)^{2}-m_{B}^{2}\right),
\end{aligned}
$$

where $m_{A}$ and $m_{B}$ denote the masses of the two heavy mesons, respectively. Then it is straight forward to carry out the integral over the four-vector $q_{\alpha}$ and obtain the twomeson scattering state contributions $\Pi_{T W}\left(p^{2}\right)$ and $\Pi_{T W}^{-}\left(p^{2}\right)$ through dispersion relation.

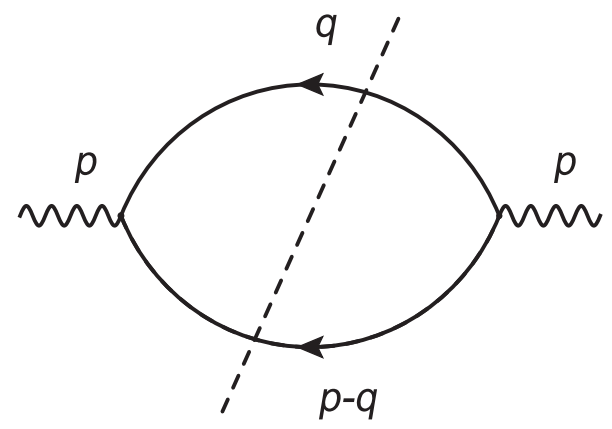

FIG. 4. The Feynman diagrams for the two-meson intermediate states, where the dashed line represents the cut.

In this paper, we carry out the operator product expansion to the vacuum condensates up to dimension-10 and take into account the vacuum condensates which are vacuum expectations of the quark-gluon operators of the $\operatorname{order} \mathcal{O}\left(\alpha_{s}^{k}\right)$ with $k \leq 1$. In calculations, we assume vacuum saturation for the higher dimensional vacuum condensates. For the current $J_{\mu}(x)$, we take into account the vacuum condensates $\langle\bar{q} q\rangle,\left\langle\frac{\alpha_{s}}{\pi} G G\right\rangle,\left\langle\bar{q} g_{s} \sigma G q\right\rangle,\langle\bar{q} q\rangle^{2}, g_{s}^{2}\langle\bar{q} q\rangle^{2}$, $\langle\bar{q} q\rangle\left\langle\frac{\alpha_{s}}{\pi} G G\right\rangle,\langle\bar{q} q\rangle\left\langle\bar{q} g_{s} \sigma G q\right\rangle,\langle\bar{q} q\rangle^{2}\left\langle\frac{\alpha_{s}}{\pi} G G\right\rangle,\left\langle\bar{q} g_{s} \sigma G q\right\rangle^{2}$. The four-quark condensate $g_{s}^{2}\langle\bar{q} q\rangle^{2}$ comes from the terms $\left\langle\bar{q} \gamma_{\mu} t^{a} q g_{s} D_{\eta} G_{\lambda \tau}^{a}\right\rangle, \quad\left\langle\bar{q}_{j} D_{\mu}^{\dagger} D_{\nu}^{\dagger} D_{\alpha}^{\dagger} q_{i}\right\rangle$, and $\left\langle\bar{q}_{j} D_{\mu} D_{\nu} D_{\alpha} q_{i}\right\rangle$, rather than comes from the perturbative corrections of the $\langle\bar{q} q\rangle^{2}$. The four-quark condensate $g_{s}^{2}\langle\bar{q} q\rangle^{2}$ plays an important role in choosing the input parameters due to the relation $g_{s}^{2}=4 \pi \alpha_{s}(\mu)$, which introduces explicit energy scale dependence; on the other hand, it plays a minor important role in numerical calculations. For the current $J_{\mu \nu}(x)$, we take into account the vacuum condensates $\langle\bar{s} s\rangle$, $\left\langle\frac{\alpha_{s}}{\pi} G G\right\rangle, \quad\left\langle\bar{s} g_{s} \sigma G s\right\rangle,\langle\bar{s} s\rangle^{2}, \quad\langle\bar{s} s\rangle\left\langle\frac{\alpha_{s}}{\pi} G G\right\rangle, \quad\langle\bar{s} s\rangle\left\langle\bar{s} g_{s} \sigma G s\right\rangle$, $\langle\bar{s} s\rangle^{2}\left\langle\frac{\alpha_{s}}{\pi} G G\right\rangle,\left\langle\bar{s} g_{s} \sigma G s\right\rangle^{2}$, and neglect the condensate $g_{s}^{2}\langle\bar{s} s\rangle^{2}$. After carrying out the operator product expansion, we obtain the analytical expressions of the correlation functions $\Pi\left(p^{2}\right)$ and $\Pi_{-}\left(p^{2}\right)$ at the quark-gluon level,

$$
\begin{aligned}
\Pi\left(p^{2}\right) & =\int_{4 m_{c}^{2}}^{s_{0}} \frac{\rho_{Z, \mathrm{QCD}}(s)}{s-p^{2}}+\cdots, \\
\Pi_{-}\left(p^{2}\right) & =\int_{4 m_{c}^{2}}^{s_{0}} \frac{\rho_{X, \mathrm{QCD}}(s)}{s-p^{2}}+\cdots,
\end{aligned}
$$

where $\rho_{Z, \mathrm{QCD}}(s)$ and $\rho_{X, \mathrm{QCD}}(s)$ are the QCD spectral densities,

$$
\begin{aligned}
\rho_{Z, \mathrm{QCD}}(s) & =\left.\frac{1}{\pi} \operatorname{Im} \Pi(s+i \epsilon)\right|_{\epsilon \rightarrow 0}, \\
\rho_{X, \mathrm{QCD}}(s) & =\left.\frac{1}{\pi} \operatorname{Im} \Pi_{-}(s+i \epsilon)\right|_{\epsilon \rightarrow 0} .
\end{aligned}
$$

According to the assertion of Lucha et al. [13], all the contributions of the order $\mathcal{O}\left(\alpha_{s}^{k}\right)$ with $k \leq 1$ are exactly canceled out by the meson-meson scattering states, and we can set 


$$
\begin{aligned}
\Pi\left(p^{2}\right) & =\Pi_{T W}\left(p^{2}\right)+\cdots, \\
\Pi_{-}\left(p^{2}\right) & =\Pi_{T W}^{-}\left(p^{2}\right)+\cdots
\end{aligned}
$$

at the hadron side, as we carry out the operator product expansion by taking into account only the contributions of the order $\mathcal{O}\left(\alpha_{s}^{k}\right)$ with $k \leq 1$. Now, let us take the quarkhadron duality below the continuum threshold $s_{0}$ and saturate the hadron side of the correlation functions with the meson-meson scattering states, then perform Borel transform with respect to the variable $P^{2}=-p^{2}$ to obtain the QCD sum rules,

$$
\begin{aligned}
& \Pi_{T W}\left(T^{2}\right)=\frac{\lambda_{D D^{*}}^{2}}{16 \pi^{2}} \int_{\Delta_{1}^{2}}^{s_{0}} d s \frac{\sqrt{\lambda\left(s, m_{D}^{2}, m_{D^{*}}^{2}\right)}}{s}\left[1+\frac{\lambda\left(s, m_{D}^{2}, m_{D^{*}}^{2}\right)}{12 s m_{D^{*}}^{2}}\right] \exp \left(-\frac{s}{T^{2}}\right) \\
& +\frac{\lambda_{D D_{0}}^{2}}{16 \pi^{2}} \int_{\Delta_{2}^{2}}^{s_{0}} d s \frac{\sqrt{\lambda\left(s, m_{D}^{2}, m_{D_{0}}^{2}\right)}}{s} \frac{\lambda\left(s, m_{D}^{2}, m_{D_{0}}^{2}\right)}{12 s} \exp \left(-\frac{s}{T^{2}}\right) \\
& =\kappa \int_{4 m_{c}^{2}}^{s_{0}} d s \rho_{\mathrm{Z}, \mathrm{QCD}}(s) \exp \left(-\frac{s}{T^{2}}\right) \text {, } \\
& \Pi_{T W}^{-}\left(T^{2}\right)=\frac{\lambda_{D_{s}^{*} D_{s 1}}^{2}}{16 \pi^{2}} \int_{\Delta_{3}^{2}}^{s_{0}} d s \frac{\sqrt{\lambda\left(s, m_{D_{s}^{*}}^{2}, m_{D_{s 1}}^{2}\right)}}{s}\left[1+\frac{\lambda\left(s, m_{D_{s}^{*}}^{2}, m_{D_{s 1}}^{2}\right)}{12 s m_{D_{s}^{*}}^{2}} \frac{\lambda\left(s, m_{D_{s}^{*}}^{2}, m_{D_{s 1}}^{2}\right)}{12 s m_{D_{s 1}}^{2}}\right] \exp \left(-\frac{s}{T^{2}}\right) \\
& +\frac{\lambda_{D_{s}^{*} D_{s}}^{2}}{16 \pi^{2}} \int_{\Delta_{4}^{2}}^{s_{0}} d s \frac{\sqrt{\lambda\left(s, m_{D_{s}^{*}}^{2}, m_{D_{s}}^{2}\right)}}{s} \frac{\lambda\left(s, m_{D_{s}^{*}}^{2}, m_{D_{s}}^{2}\right)}{12 s} \exp \left(-\frac{s}{T^{2}}\right) \\
& +\frac{\lambda_{D_{s 0} D_{s 1}}^{2}}{16 \pi^{2}} \int_{\Delta_{5}^{2}}^{s_{0}} d s \frac{\sqrt{\lambda\left(s, m_{D_{s 0}}^{2}, m_{D_{s 1}}^{2}\right)}}{s} \frac{\lambda\left(s, m_{D_{s 0}}^{2}, m_{D_{s 1}}^{2}\right)}{12 s} \exp \left(-\frac{s}{T^{2}}\right) \\
& =\kappa \int_{4 m_{c}^{2}}^{s_{0}} d s \rho_{X, \mathrm{QCD}}(s) \exp \left(-\frac{s}{T^{2}}\right)
\end{aligned}
$$

the explicit expressions of the QCD spectral densities $\rho_{Z, \mathrm{QCD}}(s)$ and $\rho_{X, \mathrm{QCD}}(s)$ are given in the Appendix, where we have rewritten the terms of the forms $\frac{d}{d s} \delta(s-$ $\left.\bar{m}_{c}^{2}\right), \frac{d^{2}}{d s^{2}} \delta\left(s-\bar{m}_{c}^{2}\right), \cdots, \frac{d}{d s} \delta\left(s-\tilde{m}_{c}^{2}\right), \frac{d^{2}}{d s^{2}} \delta\left(s-\tilde{m}_{c}^{2}\right), \cdots \quad$ in more concise forms. We saturate the QCD side of the correlation functions with the two-meson scattering states at the hadron side "by hand" according to the assertion of Lucha et al. [13]. In Sec. II, we present detailed discussions to approve that the assertion is questionable, and we have to introduce some parameters to evaluate the assertion in practical calculations. In Eqs. (32) and (33), we introduce the parameter $\kappa$ to measure the deviations from 1 ; if $\kappa \approx 1$, we can get the conclusion tentatively that the meson-meson scattering states can saturate the QCD sum rules. Then we differentiate Eqs. (32) and (33) with respect to $\frac{1}{T^{2}}$ and obtain two additional QCD sum rules,

$-\frac{d \Pi_{T W}\left(T^{2}\right)}{d\left(1 / T^{2}\right)}=-\kappa \frac{d}{d\left(1 / T^{2}\right)} \int_{4 m_{c}^{2}}^{s_{0}} d s \rho_{Z, \mathrm{QCD}}(s) \exp \left(-\frac{s}{T^{2}}\right)$,

$$
-\frac{d \Pi_{T W}^{-}\left(T^{2}\right)}{d\left(1 / T^{2}\right)}=-\kappa \frac{d}{d\left(1 / T^{2}\right)} \int_{4 m_{c}^{2}}^{s_{0}} d s \rho_{X, \mathrm{QCD}}(s) \exp \left(-\frac{s}{T^{2}}\right) .
$$

Thereafter, we will denote the QCD sum rules in Eqs. (34) and (35) as the QCDSR I and the QCD sum rules in Eqs. (32) and (33) as the QCDSR II.

On the other hand, if the meson-meson scattering states cannot saturate the QCD sum rules, we have to introduce the tetraquark molecular states to saturate the QCD sum rules,

$$
\begin{aligned}
& \lambda_{Z}^{2} \exp \left(-\frac{M_{Z}^{2}}{T^{2}}\right)=\int_{4 m_{c}^{2}}^{s_{0}} d s \rho_{Z, \mathrm{QCD}}(s) \exp \left(-\frac{s}{T^{2}}\right) \\
& \lambda_{X}^{2} \exp \left(-\frac{M_{X}^{2}}{T^{2}}\right)=\int_{4 m_{c}^{2}}^{s_{0}} d s \rho_{X, \mathrm{QCD}}(s) \exp \left(-\frac{s}{T^{2}}\right) .
\end{aligned}
$$

We differentiate Eqs. (36) and (37) with respect to $\frac{1}{T^{2}}$ and obtain two QCD sum rules for the masses of the tetraquark molecular states, 


$$
\begin{aligned}
& M_{Z}^{2}= \frac{-\frac{d}{d\left(1 / T^{2}\right)} \int_{4 m_{c}^{2}}^{s_{0}} d s \rho_{Z, \mathrm{QCD}}(s) \exp \left(-\frac{s}{T^{2}}\right)}{\int_{4 m_{c}^{2}}^{s_{0}} d s \rho_{Z, \mathrm{QCD}}(s) \exp \left(-\frac{s}{T^{2}}\right)}, \\
& M_{X}^{2}=\frac{-\frac{d}{d\left(1 / T^{2}\right)} \int_{4 m_{c}^{2}}^{s_{0}} d s \rho_{X, \mathrm{QCD}}(s) \exp \left(-\frac{s}{T^{2}}\right)}{\int_{4 m_{c}^{2}}^{s_{0}} d s \rho_{X, \mathrm{QCD}}(s) \exp \left(-\frac{s}{T^{2}}\right)} .
\end{aligned}
$$

\section{NUMERICAL RESULTS AND DISCUSSIONS}

At the QCD side, we choose the standard values of the vacuum condensates $\langle\bar{q} q\rangle=-(0.24 \pm 0.01 \mathrm{GeV})^{3},\langle\bar{s} s\rangle=$ $(0.8 \pm 0.1)\langle\bar{q} q\rangle,\left\langle\bar{q} g_{s} \sigma G q\right\rangle=m_{0}^{2}\langle\bar{q} q\rangle,\left\langle\bar{s} g_{s} \sigma G s\right\rangle=m_{0}^{2}\langle\bar{s} s\rangle$, $m_{0}^{2}=(0.8 \pm 0.1) \mathrm{GeV}^{2},\left\langle\frac{\alpha_{s} G G}{\pi}\right\rangle=(0.33 \mathrm{GeV})^{4}$ at the energy scale $\mu=1 \mathrm{GeV}$ [19-21] and choose the $\overline{\mathrm{MS}}$ masses $m_{c}\left(m_{c}\right)=(1.275 \pm 0.025) \mathrm{GeV}$ and $m_{s}(\mu=2 \mathrm{GeV})=$ $(0.095 \pm 0.005) \mathrm{GeV}$ from the Particle Data Group [2], and set $m_{u}=m_{d}=0$. Moreover, we take into account the energy-scale dependence of the input parameters,

$$
\begin{aligned}
\langle\bar{q} q\rangle(\mu) & =\langle\bar{q} q\rangle(1 \mathrm{GeV})\left[\frac{\alpha_{s}(1 \mathrm{GeV})}{\alpha_{s}(\mu)}\right]^{\frac{12}{25}}, \\
\langle\bar{s} s\rangle(\mu)= & \langle\bar{s} s\rangle(1 \mathrm{GeV})\left[\frac{\alpha_{s}(1 \mathrm{GeV})}{\alpha_{s}(\mu)}\right]^{\frac{12}{25}}, \\
\left\langle\bar{q} g_{s} \sigma G q\right\rangle(\mu)= & \left\langle\bar{q} g_{s} \sigma G q\right\rangle(1 \mathrm{GeV})\left[\frac{\alpha_{s}(1 \mathrm{GeV})}{\alpha_{s}(\mu)}\right]^{\frac{2}{25}}, \\
\left\langle\bar{s} g_{s} \sigma G s\right\rangle(\mu)= & \left\langle\bar{s} g_{s} \sigma G s\right\rangle(1 \mathrm{GeV})\left[\frac{\alpha_{s}(1 \mathrm{GeV})}{\alpha_{s}(\mu)}\right]^{\frac{2}{25}}, \\
m_{c}(\mu)= & m_{c}\left(m_{c}\right)\left[\frac{\alpha_{s}(\mu)}{\alpha_{s}\left(m_{c}\right)}\right]^{\frac{12}{25}}, \\
m_{s}(\mu)= & m_{s}(2 \mathrm{GeV})\left[\frac{\alpha_{s}(\mu)}{\alpha_{s}(2 \mathrm{GeV})}\right]^{\frac{12}{25}}, \\
\alpha_{s}(\mu)= & \frac{1}{b_{0} t}\left[1-\frac{b_{1}}{b_{0}^{2}} \frac{\log t}{t}\right. \\
& +\frac{b_{1}^{2}\left(\log ^{2} t-\log t-1\right)+b_{0} b_{2}}{b_{0}^{4} t^{2}},
\end{aligned}
$$

where $\quad t=\log \frac{\mu^{2}}{\Lambda^{2}}, \quad b_{0}=\frac{33-2 n_{f}}{12 \pi}, \quad b_{1}=\frac{153-19 n_{f}}{24 \pi^{2}}, \quad b_{2}=$ $\frac{2857-\frac{5033}{9} n_{f}+\frac{325}{27} n_{f}^{2}}{128 \pi^{3}}, \Lambda=210 \mathrm{MeV}, 292 \mathrm{MeV}$, and $332 \mathrm{MeV}$ for the flavors $n_{f}=5,4$, and 3, respectively [2,22], and evolve all the input parameters to the ideal energy scales $\mu$ with $n_{f}=4$ to extract the tetraquark molecular masses or the parameters $\kappa$. The QCD spectral densities $\rho_{Z, \mathrm{QCD}}(s)$ and $\rho_{X, \mathrm{QCD}}(s)$, and the thresholds $4 m_{c}^{2}$ depend on the energy scales $\mu$, the values of the parameters $\kappa$, masses $M_{Z / X}$, and pole residues $\lambda_{Z / X}$ extracted from the QCD sum rules in Eqs. (32)-(39) vary with the energy scales $\mu$, we should resort to some methods to choose the ideal energy scales (or pertinent energy scales) $\mu$ to extract those quantities in a consistent way.
At the hadron side, we take the hadronic parameters as $m_{D}=1.8672 \mathrm{GeV}, m_{D_{s}}=1.9690 \mathrm{GeV}, m_{D^{*}}=$ $2.0086 \mathrm{GeV}, \quad m_{D_{s}^{*}}=2.1122 \mathrm{GeV}, \quad m_{D_{0}}=2.3245 \mathrm{GeV}$, $m_{D_{s 0}}=2.3180 \mathrm{GeV}, m_{D_{s 1}}=2.5352 \mathrm{GeV}$ from the Particle Data Group [2]; $f_{D}=0.208 \mathrm{GeV}, f_{D_{s}}=0.240 \mathrm{GeV}$, $f_{D^{*}}=0.263 \mathrm{GeV}, f_{D_{s}^{*}}=0.308 \mathrm{GeV}, f_{D_{0}}=0.373 \mathrm{GeV}$, $f_{D_{s 0}}=0.333 \mathrm{GeV}$ [23], $f_{D_{s 1}}=0.364 \mathrm{GeV}$ from the QCD sum rules.

The $D \bar{D}^{*}+D^{*} \bar{D}$ and $D_{s}^{*} \bar{D}_{s 1}-D_{s 1} \bar{D}_{s}^{*}$ thresholds are $m_{D}+m_{D^{*}}=3.88 \mathrm{GeV}$ and $m_{D_{s}^{*}}+m_{D_{s 1}}=4.65 \mathrm{GeV}$, respectively. For the conventional heavy mesons, the mass gaps between the ground states and the first radial excited states are about $0.4-0.6 \mathrm{GeV}$, so the continuum threshold parameters can be chosen as $\sqrt{s_{0}}=4.40 \pm 0.10$ and $5.15 \pm 0.10 \mathrm{GeV}$, respectively.

We search for the acceptable Borel parameters $T^{2}$ to warrant convergence of the operator product expansion and pole dominance via trial and error. First, let us define the pole contributions $\mathrm{PC}$,

$$
\mathrm{PC}=\frac{\int_{4 m_{c}^{2}}^{s_{0}} d s \rho_{Z / X, \mathrm{QCD}}(s) \exp \left(-\frac{s}{T^{2}}\right)}{\int_{4 m_{c}^{2}}^{\infty} d s \rho_{Z / X, \mathrm{QCD}}(s) \exp \left(-\frac{s}{T^{2}}\right)},
$$

and the contributions of the vacuum condensates $D(n)$,

$$
D(n)=\frac{\int_{4 m_{c}^{2}}^{s_{0}} d s \rho_{Z / X, \mathrm{QCD} ; n}(s) \exp \left(-\frac{s}{T^{2}}\right)}{\int_{4 m_{c}^{2}}^{s_{0}} d s \rho_{Z / X, \mathrm{QCD}}(s) \exp \left(-\frac{s}{T^{2}}\right)},
$$

where the subscript $n$ in the QCD spectral densities $\rho_{Z / X, \mathrm{QCD} ; n}(s)$ represents the vacuum condensates of dimension $n$.

In Fig. 5, we plot the pole contributions with variations of the energy scales of the QCD spectral densities with the parameters $T_{Z}^{2}=2.9 \mathrm{GeV}^{2}, \sqrt{s_{Z}^{0}}=4.40 \mathrm{GeV}$, and $T_{X}^{2}=$ $3.9 \mathrm{GeV}^{2}, \sqrt{s_{X}^{0}}=5.15 \mathrm{GeV}$ for the QCD spectral densities $\rho_{Z, \mathrm{QCD}}(s)$ and $\rho_{X, \mathrm{QCD}}(s)$, respectively. We choose

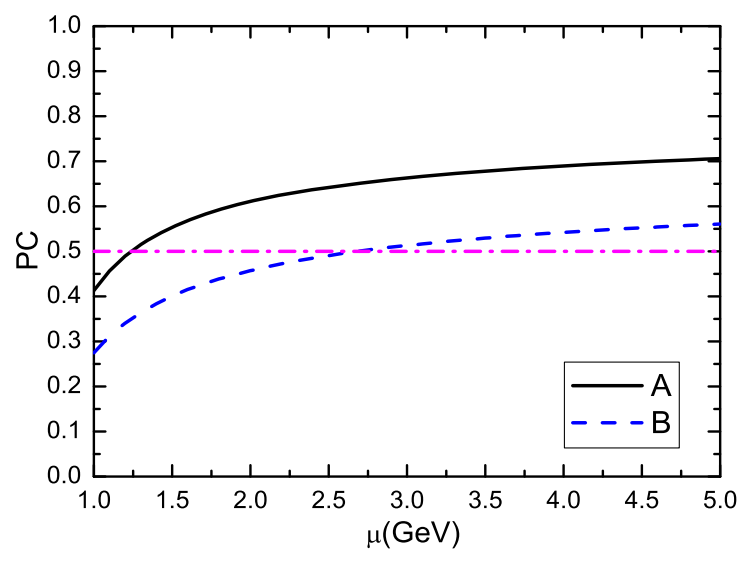

FIG. 5. The pole contributions with variations of the energy scales $\mu$, where $A$ and $B$ correspond to the QCD spectral densities $\rho_{Z, \mathrm{QCD}}(s)$ and $\rho_{X, \mathrm{QCD}}(s)$, respectively. 


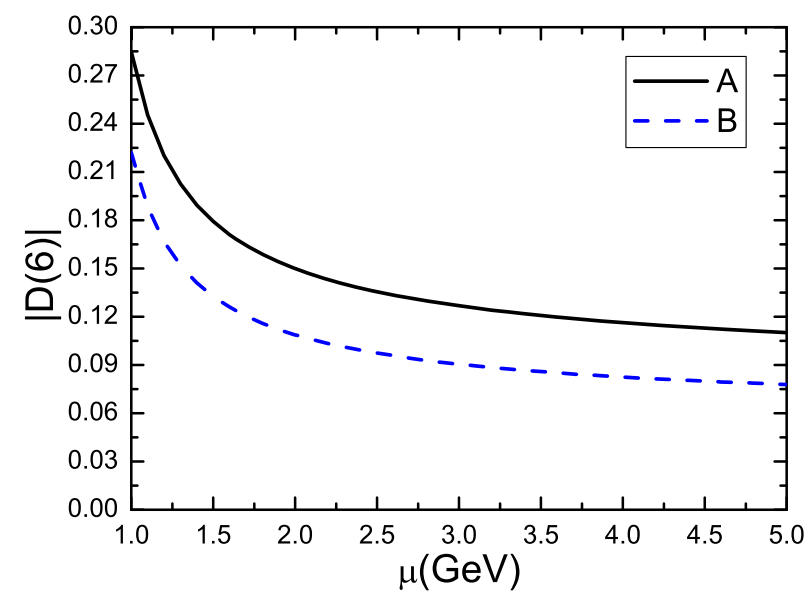

FIG. 6. The absolute values of $D(6)$ with variations of the energy scales $\mu$, where $A$ and $B$ correspond to the QCD spectral densities $\rho_{Z, \mathrm{QCD}}(s)$ and $\rho_{X, \mathrm{QCD}}(s)$, respectively.

those typical values because the continuum threshold parameters $s_{0}$ and Borel parameters $T^{2}$ have the relation $\frac{s_{Z}^{0}}{T_{Z}^{2}}=\frac{s_{X}^{0}}{T_{X}^{2}}$, the weight functions $\exp \left(-\frac{s}{T^{2}}\right)$ have the same values. From Fig. 5, we can see that the pole contributions increase monotonically and considerably with the increase of the energy scales at the region $\mu<3.0 \mathrm{GeV}$, then the pole contributions increase monotonically but slowly with the increase of the energy scales. The pole contributions exceed $50 \%$ at the energy scales $\mu=1.3$ and $2.7 \mathrm{GeV}$ for the QCD spectral densities $\rho_{Z, \mathrm{QCD}}(s)$ and $\rho_{X, \mathrm{QCD}}(s)$, respectively.

In Fig. 6, we plot the absolute values of $D(6)$ with variations of the energy scales $\mu$ of the QCD spectral densities with the parameters $T_{Z}^{2}=2.9 \mathrm{GeV}^{2}, \sqrt{s_{Z}^{0}}=$ $4.40 \mathrm{GeV}$ and $T_{X}^{2}=3.9 \mathrm{GeV}^{2}, \sqrt{s_{X}^{0}}=5.15 \mathrm{GeV}$ for the QCD spectral densities $\rho_{Z, \mathrm{QCD}}(s)$ and $\rho_{X, \mathrm{QCD}}(s)$, respectively. The contributions of the vacuum condensates of dimension 6 play a very important role in the QCD sum rules for the hidden-charm or hidden-bottom tetraquark (molecular) states. From Fig. 6, we can see that the contributions $|D(6)|$ decrease monotonically with the increase of the energy scales. A larger energy scale $\mu$ leads to a larger pole contribution, but a smaller contribution of the vacuum condensate $D(6)$. Too small contributions of the vacuum condensates will impair the stability of the QCD sum rules.

\section{A. Meson-meson scattering states alone}

We saturate the hadron side of the QCD sum rules with the meson-meson scattering states alone and study the QCD sum rues shown in Eqs. (32)-(35). In this paper, we choose the pole contributions as large as (40\%-60\%), the pole dominance criterion is satisfied. The Borel windows, continuum threshold parameters, energy scales of the QCD spectral densities, and pole contributions are shown explicitly in Table I. In the Borel windows, the contributions of the higher dimensional vacuum condensates are $|D(8)|=(3-5) \%, \quad|D(10)| \ll 1 \%$ and $D(8)=(1-2) \%$, $D(10) \ll 1 \%$ for the QCD spectral densities $\rho_{Z, \mathrm{QCD}}(s)$ and $\rho_{X, \mathrm{QCD}}(s)$, respectively. The operator product expansion converges very good.

We take into account all uncertainties of the input parameters at the QCD side and obtain the values of the $\kappa$ from the QCDSR I and II directly, which are shown in Table I. In calculations, we add an uncertainty $\delta \mu=$ $\pm 0.1 \mathrm{GeV}$ to the energy scales $\mu$. From Table I, we can see that the values $\kappa_{\mathrm{I}}=1.55 \pm 0.40$ and $\kappa_{\mathrm{II}}=1.37 \pm 0.40$ overestimate the contributions of the $\bar{u} c \bar{c} d$ meson-meson scattering states with $J^{P C}=1^{+-}$, while the values $\kappa_{\mathrm{I}}=$ $0.50 \pm 0.09$ and $\kappa_{\text {II }}=0.46 \pm 0.09$ underestimate the contributions of the $\bar{s} c \bar{c} s$ meson-meson scattering states with $J^{P C}=1^{-+}$. In the two cases, the values of $\kappa$ from QCDSR I and II deviate from 1 significantly, the two-meson scattering sates cannot saturate the QCD sum rules.

In Fig. 7, we plot the values of $\kappa$ with variations of the Borel parameters $T^{2}$ with the continuum threshold parameters $\sqrt{s_{0}}=4.40$ and $5.15 \mathrm{GeV}$ for the $\bar{u} c \bar{c} d$ and $\bar{s} c \bar{c} s$ meson-meson scattering states, respectively, where we normalize the values of $\kappa$ to be 1 at the points $T^{2}=1.5$ and $2.4 \mathrm{GeV}^{2}$ for the $\bar{u} c \bar{c} d$ and $\bar{s} c \bar{c} s$ meson-meson scattering states, respectively. In this way, we can see the variation trends of $\kappa$ with the changes of the Borel parameters more explicitly. From Fig. 7, we can see that the values of $\kappa$ increase monotonically and quickly with the increase of the Borel parameters $T^{2}$, no platform appears, which indicates that the QCD sum rules in Eqs. (32) and (33) obtained according to the assertion of Lucha et al. are unreasonable. Reasonable QCD sum rules lead to platforms flat enough or not flat enough, rather than no evidence of platforms.

Now, we can obtain the conclusion tentatively that the meson-meson scattering states cannot saturate the QCD sum rules at the hadron side.

TABLE I. The Borel parameters, continuum threshold parameters, energy scales of the QCD spectral densities, pole contributions and $\kappa$ for the QCDSR I and II, where we show the quark constituents of the meson-meson scattering states in the brackets.

\begin{tabular}{lcccccc}
\hline \hline$J^{P C}$ & $T^{2}\left(\mathrm{GeV}^{2}\right)$ & $\sqrt{s_{0}}(\mathrm{GeV})$ & $\mu(\mathrm{GeV})$ & Pole & $\kappa_{\mathrm{I}}$ & $\kappa_{\mathrm{II}}$ \\
\hline $1^{+-}(\bar{u} c \bar{c} d)$ & $2.7-3.1$ & $4.40 \pm 0.10$ & 1.3 & $(40-63) \%$ & $1.55 \pm 0.40$ & $1.37 \pm 0.40$ \\
$1^{-+}(\bar{s} c \bar{c} s)$ & $3.7-4.1$ & $5.15 \pm 0.10$ & 2.9 & $(42-60) \%$ & $0.50 \pm 0.09$ & $0.46 \pm 0.09$ \\
\hline \hline
\end{tabular}



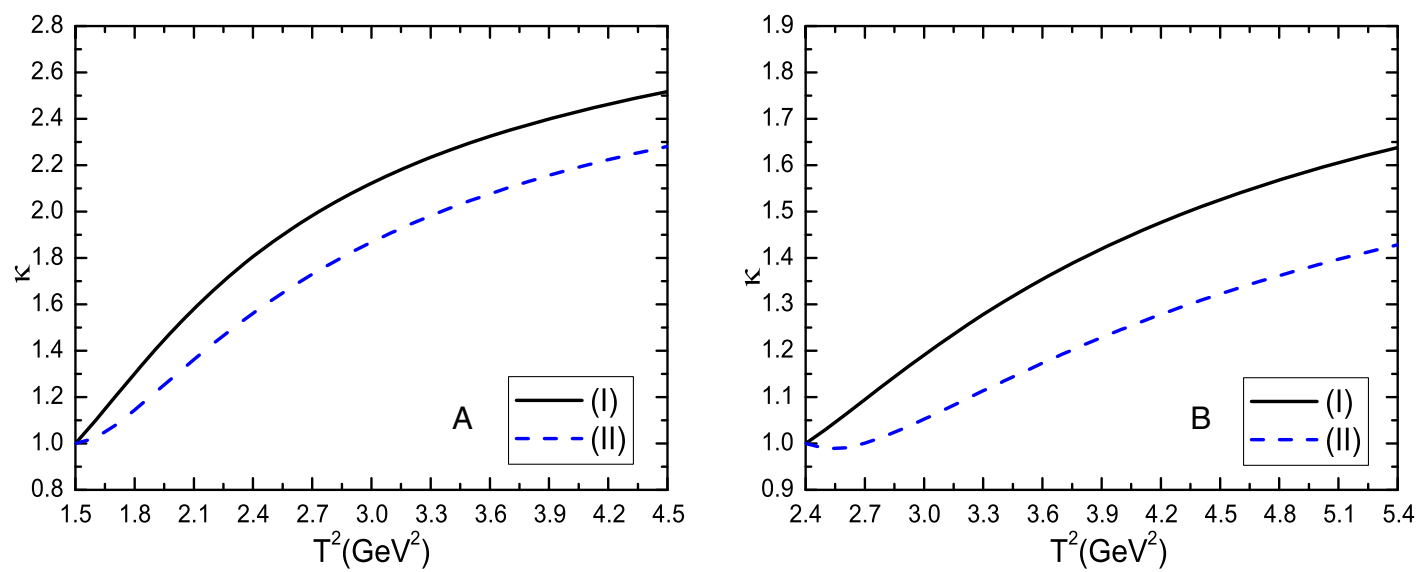

FIG. 7. The $\kappa$ with variations of the Borel parameters $T^{2}$, where $A$ and $B$ correspond to the $\bar{u} c \bar{c} d$ and $\bar{s} c \bar{c} s$ meson-meson scattering states, respectively, (I) and (II) correspond to QCDSR I and II, respectively, and the $\kappa$ values are normalized to be 1 for the Borel parameters $T^{2}=1.5$ and $2.4 \mathrm{GeV}^{2}$, respectively.

\section{B. Tetraquark molecular states alone}

We saturate the hadron side of the QCD sum rules with the tetraquark molecular states alone and study the QCD sum rues shown in Eqs. (36)-(39).

In Fig. 8, we plot the masses with variations of the energy scales of the QCD spectral densities with the parameters $T_{Z}^{2}=2.9 \mathrm{GeV}^{2}, \sqrt{s_{Z}^{0}}=4.40 \mathrm{GeV}$ and $T_{X}^{2}=$ $3.9 \mathrm{GeV}^{2}, \sqrt{s_{X}^{0}}=5.15 \mathrm{GeV}$ for $\bar{u} c \bar{c} d$ and $\bar{s} c \bar{c} s$ tetraquark molecular states, respectively. From Fig. 8, we can see that the values of the masses decrease monotonically and slowly with the increase of the energy scales $\mu$. Now, we encounter the problem how to choose the pertinent energy scales of the QCD spectral densities $\rho_{Z, \mathrm{QCD}}(s)$ and $\rho_{X, \mathrm{QCD}}(s)$.

We describe the heavy tetraquark system $Q \bar{Q} q \bar{q}$ (or the exotic $X, Y, Z$ states) by a double-well potential with the two light quarks $q$ and $\bar{q}$ lying in the two potential wells,

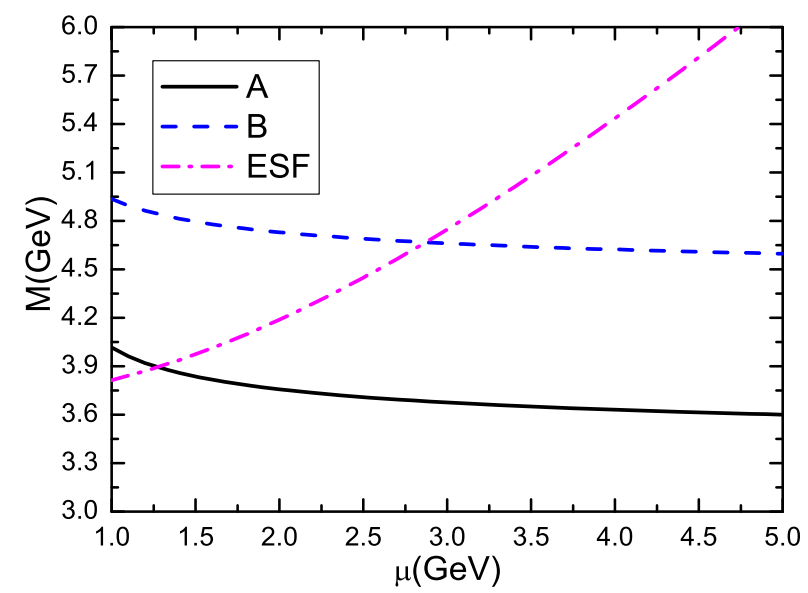

FIG. 8. The masses with variations of the energy scales $\mu$, where $A$ and $B$ correspond to the $\bar{u} c \bar{c} d$ and $\bar{s} c \bar{c} s$ tetraquark molecular states, respectively; the ESF denotes the energy scale formula $M_{X / Z}=\sqrt{\mu^{2}+4 \times(1.84 \mathrm{GeV})^{2}}$. respectively. In the heavy quark limit, the $Q$ quark serves as a static well potential and attracts the light quark $q$ to form a diquark in the color antitriplet channel or attracts the light antiquark $\bar{q}$ to form a meson in the color-singlet channel. Then the heavy tetraquark (molecular) states are characterized by the effective heavy quark mass $\mathbb{M}_{Q}$ (or constituent quark mass) and the virtuality $V=$ $\sqrt{M_{X / Y / Z}^{2}-\left(2 \mathbb{M}_{Q}\right)^{2}}$. It is natural to choose the energy scales of the QCD spectral densities as

$$
\mu^{2}=V^{2}=M_{X / Y / Z}^{2}-\left(2 \mathbb{M}_{Q}\right)^{2} .
$$

Analysis of $J / \psi$ and $\Upsilon$ with the famous Coulomb-pluslinear potential or Cornell potential leads to the constituent quark masses $m_{c}=1.84 \mathrm{GeV}$ and $m_{b}=5.17 \mathrm{GeV}$ [24]. If we set $\mathbb{M}_{c}=m_{c}=1.84 \mathrm{GeV}$, we can obtain the dashdotted line $M_{X / Z}=\sqrt{\mu^{2}+4 \times(1.84 \mathrm{GeV})^{2}}$ in Fig. 8, which intersects with the lines of the masses of the $\bar{u} c \bar{c} d$ and $\bar{s} c \bar{c} s$ tetraquark molecular states at the energy scales about $\mu=1.3$ and $2.9 \mathrm{GeV}$, respectively. The values of the cross points are $3.89 \mathrm{GeV}$ and $4.67 \mathrm{GeV}$, which happen to coincide with $D \bar{D}^{*}+D^{*} \bar{D}$ and $D_{s}^{*} \bar{D}_{s 1}-D_{s 1} \bar{D}_{s}^{*}$ thresholds 3.88 and $4.65 \mathrm{GeV}$, respectively. The old value $\mathbb{M}_{c}=1.84 \mathrm{GeV}$ and updated value $\mathbb{M}_{c}=1.85 \mathrm{GeV}$ fitted in the QCD sum rules for the hidden-charm tetraquark molecular states are all consistent with the constituent quark mass $m_{c}=1.84 \mathrm{GeV}[11,12]$. We can set the value of the effective $c$-quark mass as $\mathbb{M}_{c}=1.84 \pm 0.01 \mathrm{GeV}$.

In this paper, we use the energy scale formula $\mu=$ $\sqrt{M_{X / Z}^{2}-4 \times(1.84 \mathrm{GeV})^{2}}$ as the constraints to choose the best energy scales of the QCD spectral densities.

Again, we choose the pole contributions as large as (40\%-60\%). The Borel windows, continuum threshold parameters, energy scales of the QCD spectral densities, 
TABLE II. The Borel windows, continuum threshold parameters, energy scales of the QCD spectral densities, pole contributions, masses and pole residues of the $\bar{u} c \bar{c} d$ and $\bar{s} c \bar{c} s$ tetraquark molecular states.

\begin{tabular}{lcccccc}
\hline \hline$J^{P C}$ & $T^{2}\left(\mathrm{GeV}^{2}\right)$ & $\sqrt{s_{0}}(\mathrm{GeV})$ & $\mu(\mathrm{GeV})$ & Pole & $M(\mathrm{GeV})$ & $\lambda\left(10^{-2} \mathrm{GeV}^{5}\right)$ \\
\hline $1^{+-}(\bar{u} c \bar{c} d)$ & $2.7-3.1$ & $4.40 \pm 0.10$ & 1.3 & $(40-63) \%$ & $3.89 \pm 0.09$ & $1.72 \pm 0.30$ \\
$1^{-+}(\bar{s} c \bar{c} s)$ & $3.7-4.1$ & $5.15 \pm 0.10$ & 2.9 & $(42-60) \%$ & $4.67 \pm 0.08$ & $6.87 \pm 0.84$ \\
\hline \hline
\end{tabular}

and pole contributions are shown explicitly in Table II, just like in Table I. Again, in the Borel windows, $|D(8)|=$ $(3-5) \%,|D(10)| \ll 1 \%$ and $D(8)=(1-2) \%, D(10) \ll$ $1 \%$ for the QCD spectral densities $\rho_{Z, \mathrm{QCD}}(s)$ and $\rho_{X, \mathrm{QCD}}(s)$, respectively.

Now, let us take into account all uncertainties of the input parameters and obtain the values of the masses and pole residues of the tetraquark molecular states, which are shown in Table II and Figs. 9 and 10. In calculations, we add an uncertainty $\delta \mu= \pm 0.1 \mathrm{GeV}$ to the energy scales $\mu$ according to the uncertainty in the effective $c$-quark mass $\mathbb{M}_{c}=1.84 \pm 0.01 \mathrm{GeV}$. From Figs. 9 and 10 , we can see that there appear Borel platforms in the Borel windows indeed. The tetraquark molecular states alone can satisfy the QCD sum rules.

\section{Taking into account the meson-meson scattering states besides the tetraquark molecular states}

From the previous two subsections, we observe that the meson-meson scattering states alone cannot saturate the QCD sum rules, while the tetraquark molecular states alone can saturate the QCD sum rules. However, the quantum field theory does not forbid the couplings between the

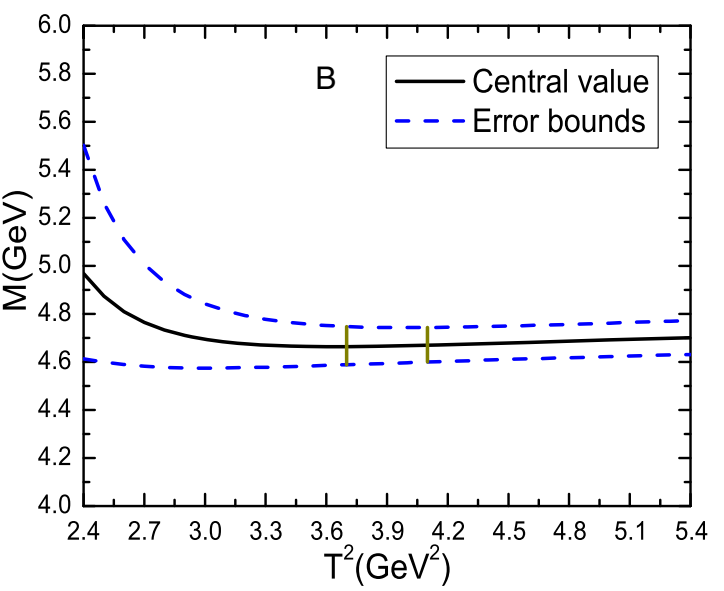

FIG. 9. The masses with variations of the Borel parameters $T^{2}$, where $A$ and $B$ correspond to the $\bar{u} c \bar{c} d$ and $\bar{s} c \bar{c} s$ tetraquark molecular states, respectively; the regions between the two vertical lines are the Borel windows.
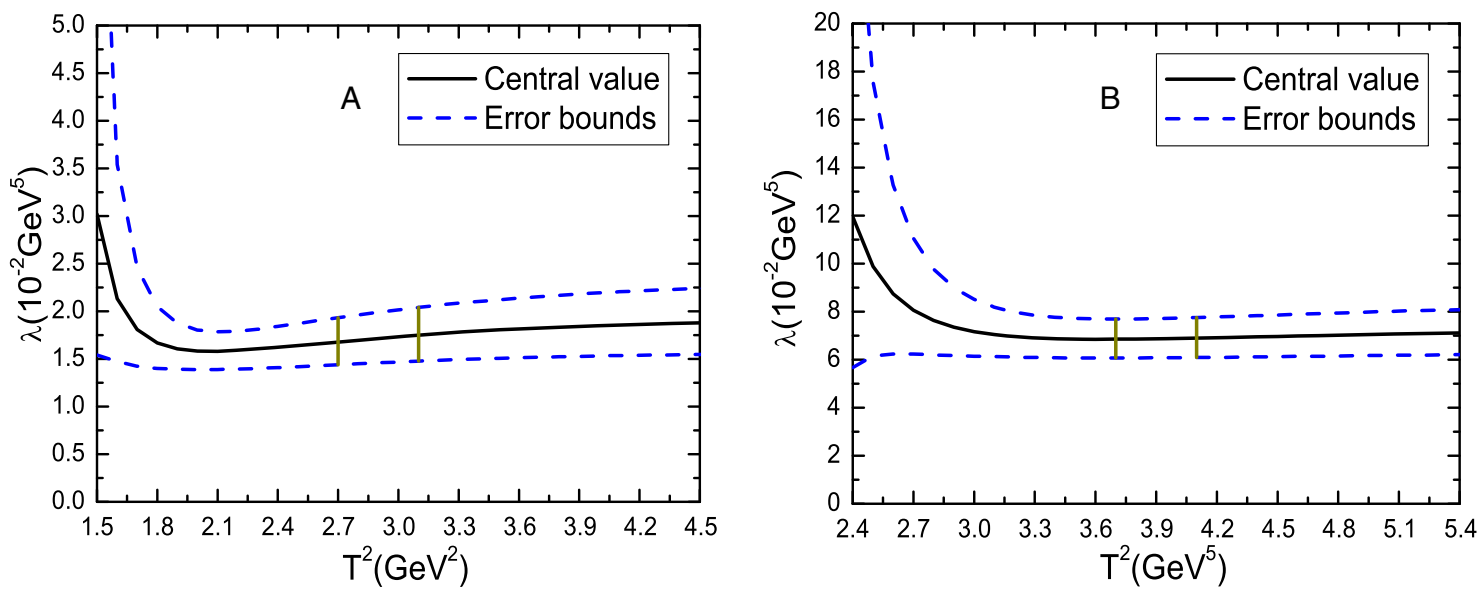

FIG. 10. The pole residues with variations of the Borel parameters $T^{2}$, where $A$ and $B$ correspond to the $\bar{u} c \bar{c} d$ and $\bar{s} c \bar{c} s$ tetraquark molecular states, respectively, the regions between the two vertical lines are the Borel windows. 
four-quark currents and meson-meson scattering states if they have the same quantum numbers. We should take into account both the tetraquark molecular states and the mesonmeson scattering states at the hadron side.
Now, we study the contributions of the intermediate meson-meson scattering states $D \bar{D}^{*}, J / \psi \pi, J / \psi \rho$, etc., besides the tetraquark molecular state $Z_{c}$ to the correlation function $\Pi_{\mu \nu}(p)$ as an example,

$$
\Pi_{\mu \nu}(p)=-\frac{\hat{\lambda}_{Z}^{2}}{p^{2}-\hat{M}_{Z}^{2}-\Sigma_{D D^{*}}\left(p^{2}\right)-\Sigma_{J / \psi \pi}\left(p^{2}\right)-\Sigma_{J / \psi \rho}\left(p^{2}\right)+\cdots} \tilde{g}_{\mu \nu}(p)+\cdots,
$$

where $\tilde{g}_{\mu \nu}(p)=-g_{\mu \nu}+\frac{p_{\mu} p_{\nu}}{p^{2}}$. We choose the bare quantities $\hat{\lambda}_{Z}$ and $\hat{M}_{Z}$ to absorb the divergences in the self-energies $\Sigma_{D \bar{D}^{*}}\left(p^{2}\right), \Sigma_{J / \psi \pi}\left(p^{2}\right), \Sigma_{J / \psi \rho}\left(p^{2}\right)$, etc. The renormalized energies satisfy the relation $p^{2}-M_{Z}^{2}-\bar{\Sigma}_{D D^{*}}\left(p^{2}\right)-$ $\bar{\Sigma}_{J / \psi \pi}\left(p^{2}\right)-\bar{\Sigma}_{J / \psi \rho}\left(p^{2}\right)+\cdots=0$, where the overlines above the self-energies denote that the divergent terms have been subtracted. As the tetraquark molecular state $Z_{c}$ is unstable, the relation should be modified, $p^{2}-M_{Z}^{2}-\operatorname{Re} \bar{\Sigma}_{D D^{*}}\left(p^{2}\right)-$ $\operatorname{Re} \bar{\Sigma}_{J / \psi \pi}\left(p^{2}\right)-\operatorname{Re} \bar{\Sigma}_{J / \psi \rho}\left(p^{2}\right)+\cdots=0$, and $-\operatorname{Im} \bar{\Sigma}_{D D^{*}}\left(p^{2}\right)-$ $\operatorname{Im} \bar{\Sigma}_{J / \psi \pi}\left(p^{2}\right)-\operatorname{Im} \bar{\Sigma}_{J / \psi \rho}\left(p^{2}\right)+\cdots=\sqrt{p^{2}} \Gamma\left(p^{2}\right)$. The negative sign in front of the self-energies comes from the special definitions in this paper; if we redefine the selfenergies, $\Sigma\left(p^{2}\right) \rightarrow-\Sigma\left(p^{2}\right)$ in Eq. (44), the negative sign can be removed. The renormalized self-energies contribute a finite imaginary part to modify the dispersion relation,

$\Pi_{\mu \nu}(p)=-\frac{\lambda_{Z}^{2}}{p^{2}-M_{Z}^{2}+i \sqrt{p^{2}} \Gamma\left(p^{2}\right)} \tilde{g}_{\mu \nu}(p)+\cdots$.
If we assign $Z_{c}(3900)$ to be the $D \bar{D}^{*}+D^{*} \bar{D}$ tetraquark molecular state with $J^{P C}=1^{+-}[11]$, the physical width $\Gamma_{Z_{c}(3900)}\left(M_{Z}^{2}\right)=(28.2 \pm 2.6) \mathrm{MeV}$ from the Particle Data Group [2].

We can take into account the finite width effect by the following simple replacement of the hadronic spectral density:

$$
\lambda_{Z}^{2} \delta\left(s-M_{Z}^{2}\right) \rightarrow \lambda_{Z}^{2} \frac{1}{\pi} \frac{M_{Z} \Gamma_{Z}(s)}{\left(s-M_{Z}^{2}\right)^{2}+M_{Z}^{2} \Gamma_{Z}^{2}(s)},
$$

where

$$
\Gamma_{Z}(s)=\Gamma_{Z} \frac{M_{Z}}{\sqrt{s}} \sqrt{\frac{s-\left(M_{D}+M_{D^{*}}\right)^{2}}{M_{Z}^{2}-\left(M_{D}+M_{D^{*}}\right)^{2}}} .
$$

Then the hadron sides of the QCD sum rules in Eqs. (36) and (38) undergo the following changes:

$$
\begin{aligned}
\lambda_{Z}^{2} \exp \left(-\frac{M_{Z}^{2}}{T^{2}}\right) & \rightarrow \lambda_{Z}^{2} \int_{\left(m_{D}+m_{D^{*}}\right)^{2}}^{s_{0}} d s \frac{1}{\pi} \frac{M_{Z} \Gamma_{Z}(s)}{\left(s-M_{Z}^{2}\right)^{2}+M_{Z}^{2} \Gamma_{Z}^{2}(s)} \exp \left(-\frac{s}{T^{2}}\right), \\
& =(0.78 \sim 0.79) \lambda_{Z}^{2} \exp \left(-\frac{M_{Z}^{2}}{T^{2}}\right) \\
\lambda_{Z}^{2} M_{Z}^{2} \exp \left(-\frac{M_{Z}^{2}}{T^{2}}\right) & \rightarrow \lambda_{Z}^{2} \int_{\left(m_{D}+m_{D^{*}}\right)^{2}}^{s_{0}} d s s \frac{1}{\pi} \frac{M_{Z} \Gamma_{Z}(s)}{\left(s-M_{Z}^{2}\right)^{2}+M_{Z}^{2} \Gamma_{Z}^{2}(s)} \exp \left(-\frac{s}{T^{2}}\right), \\
& =(0.80 \sim 0.81) \lambda_{Z}^{2} M_{Z}^{2} \exp \left(-\frac{M_{Z}^{2}}{T^{2}}\right)
\end{aligned}
$$

with the value $\sqrt{s_{0}}=4.40 \mathrm{GeV}$. We can absorb the numerical factors $0.78 \sim 0.79$ and $0.80 \sim 0.81$ into the pole residue with the simple replacement $\lambda_{Z} \rightarrow 0.89 \lambda_{Z}$ safely, the intermediate meson loops cannot affect the mass $M_{Z}$ significantly, but affect the pole residue remarkably, which are consistent with the fact that we obtain the masses of the tetraquark molecular states from a fraction; see Eqs. (38) and (39). If we only take into account the tetraquark molecular states at the hadron side, we can obtain reasonable molecule masses but overestimate the pole residues.

\section{CONCLUSION}

The quarks and gluons are confined objects; they cannot be put on the mass shell. It is questionable to use the Landau equation to study the quark-gluon bound states. Furthermore, we carry out the operator product expansion in the deep Euclidean region $p^{2} \rightarrow-\infty$ in the QCD sum rules, where the Landau singularities cannot exist. If we insist on applying the Landau equation to study the Feynman diagrams in the QCD sum rules, we should choose the pole masses rather than the $\overline{\mathrm{MS}}$ masses, which 
lead to obvious problems in the QCD sum rules for the traditional or normal charmonium and bottomonium states.

Lucha et al. assert that the contributions at the order $\mathcal{O}\left(\alpha_{s}^{k}\right)$ with $k \leq 1$ in the operator product expansion, which are factorizable in the color space, are exactly canceled out by the meson-meson scattering states, the nonfactorizable diagrams in the color space, if have a Landau singularity, begin to make contributions to the tetraquark (molecular) states, the tetraquark molecular states begin to receive contributions at the order $\mathcal{O}\left(\alpha_{s}^{2}\right)$. In fact, the nonactorizable Feynman diagrams begin to appear at the order $\mathcal{O}\left(\alpha_{s}^{0} / \alpha_{s}^{1}\right)$ rather than at the order $\mathcal{O}\left(\alpha_{s}^{2}\right)$ and make contributions to the tetraquark molecular states. Furthermore, the Landau singularities obtained by Lucha et al. are questionable, as the Landau singularities appear at the region $p^{2} \geq\left(\hat{m}_{u / s}+\right.$ $\left.\hat{m}_{d / s}+\hat{m}_{c}+\hat{m}_{c}\right)^{2}$ rather than at the deep Euclidean region $p^{2} \rightarrow-\infty$.

The meson-meson scattering state and tetraquark molecular state both have four valence quarks, which form two color-neutral clusters; we cannot distinguish which Feynman diagrams contribute to the meson-meson scattering state or tetraquark molecular state based on the two color-neutral clusters in the factorizable Feynman diagrams. The Landau equation servers as a kinematical equation in the momentum space and is independent on the factorizable and nonfactorizable properties of the Feynman diagrams in the color space.

We choose the axial-vector current $J_{\mu}(x)$ and tensor current $J_{\mu \nu}(x)$ to examine the outcome if the assertion of
Lucha et al. is right. The axial-vector current $J_{\mu}(x)$ couples potentially to the charged $D \bar{D}^{*}+D^{*} \bar{D}$ meson-meson scattering states or tetraquark molecular states with $J^{P C}=1^{+-}$, while the tensor current $J_{\mu \nu}(x)$ couples potentially to the neutral $D_{s}^{*} \bar{D}_{s 1}-D_{s 1} \bar{D}_{s}^{*}$ meson-meson scattering states or tetraquark molecular states with $J^{P C}=1^{-+}$. The quantum numbers of $D \bar{D}^{*}+D^{*} \bar{D}$ and $D_{s}^{*} \bar{D}_{s 1}-$ $D_{s 1} \bar{D}_{s}^{*}$ differ from the traditional or normal mesons significantly and are good subjects to study the exotic states. After a detailed analysis, we observe that the mesonmeson scattering states cannot saturate the QCD sum rules, while the tetraquark molecular states can saturate the QCD sum rules. We can take into account the meson-meson scattering states reasonably by adding a finite width to the tetraquark molecular states.

The Landau equation is useless to study the Feynman diagrams in the QCD sum rules for the tetraquark molecular states; the tetraquark molecular states begin to receive contributions at the order $\mathcal{O}\left(\alpha_{s}^{0} / \alpha_{s}^{1}\right)$ rather than at the order $\mathcal{O}\left(\alpha_{s}^{2}\right)$.

\section{ACKNOWLEDGMENTS}

This work is supported by National Natural Science Foundation, Grant No. 11775079.

\section{APPENDIX}

The explicit expressions of the QCD spectral densities $\rho_{Z, \mathrm{QCD}}(s)$ and $\rho_{X, \mathrm{QCD}}(s)$,

$$
\begin{gathered}
\rho_{Z, \mathrm{QCD}}(s)=\rho_{0}^{Z}(s)+\rho_{3}^{Z}(s)+\rho_{4}^{Z}(s)+\rho_{5}^{Z}(s)+\rho_{6}^{Z}(s)+\rho_{7}^{Z}(s)+\rho_{8}^{Z}(s)+\rho_{10}^{Z}(s), \\
\rho_{X, \mathrm{QCD}}(s)=\rho_{0}^{X}(s)+\rho_{3}^{X}(s)+\rho_{4}^{X}(s)+\rho_{5}^{X}(s)+\rho_{6}^{X}(s)+\rho_{7}^{X}(s)+\rho_{8}^{X}(s)+\rho_{10}^{X}(s), \\
\rho_{0}^{Z}(s)=\frac{1}{4096 \pi^{6}} \int d y d z y z(1-y-z)^{3}\left(s-\bar{m}_{c}^{2}\right)^{2}\left(35 s^{2}-26 s \bar{m}_{c}^{2}+3 \bar{m}_{c}^{4}\right), \\
\rho_{3}^{Z}(s)=-\frac{3 m_{c}\langle\bar{q} q\rangle}{256 \pi^{4}} \int d y d z(y+z)(1-y-z)\left(s-\bar{m}_{c}^{2}\right)\left(7 s-3 \bar{m}_{c}^{2}\right), \\
\rho_{4}^{Z}(s)=-\frac{m_{c}^{2}}{3072 \pi^{4}}\left\langle\frac{\alpha_{s} G G}{\pi}\right\rangle \int d y d z\left(\frac{z}{y^{2}}+\frac{y}{z^{2}}\right)(1-y-z)^{3}\left[8 s-3 \bar{m}_{c}^{2}+s^{2} \delta\left(s-\bar{m}_{c}^{2}\right)\right] \\
+\frac{1}{1024 \pi^{4}}\left\langle\frac{\alpha_{s} G G}{\pi}\right\rangle \int d y d z(y+z)(1-y-z)^{2} s\left(5 s-4 \bar{m}_{c}^{2}\right), \\
\rho_{5}^{Z}(s)=\frac{3 m_{c}\left\langle\bar{q} g_{s} \sigma G q\right\rangle}{512 \pi^{4}} \int d y d z(y+z)\left(5 s-3 \bar{m}_{c}^{2}\right) \\
-\frac{3 m_{c}\left\langle\bar{q} g_{s} \sigma G q\right\rangle}{256 \pi^{4}} \int d y d z\left(\frac{y}{z}+\frac{z}{y}\right)(1-y-z)\left(2 s-\bar{m}_{c}^{2}\right),
\end{gathered}
$$




$$
\begin{aligned}
& \rho_{6}^{Z}(s)=\frac{m_{c}^{2}\langle\bar{q} q\rangle^{2}}{16 \pi^{2}} \int d y+\frac{g_{s}^{2}\langle\bar{q} q\rangle^{2}}{864 \pi^{4}} \int d y d z y z\left[8 s-3 \bar{m}_{c}^{2}+s^{2} \delta\left(s-\bar{m}_{c}^{2}\right)\right] \\
& -\frac{g_{s}^{2}\langle\bar{q} q\rangle^{2}}{576 \pi^{4}} \int d y d z\left(\frac{z}{y}+\frac{y}{z}\right)(1-y-z)\left(7 s-4 \bar{m}_{c}^{2}\right) \\
& -\frac{m_{c}^{2} g_{s}^{2}\langle\bar{q} q\rangle^{2}}{1728 \pi^{4}} \int d y d z\left(\frac{z}{y^{2}}+\frac{y}{z^{2}}\right)(1-y-z)\left[7+5 s \delta\left(s-\bar{m}_{c}^{2}\right)\right] \\
& +\frac{g_{s}^{2}\langle\bar{q} q\rangle^{2}}{1728 \pi^{4}} \int d y d z(y+z)(1-y-z)\left(4 s-3 \bar{m}_{c}^{2}\right) \text {, } \\
& \rho_{7}^{Z}(s)=\frac{m_{c}^{3}\langle\bar{q} q\rangle}{768 \pi^{2}}\left\langle\frac{\alpha_{s} G G}{\pi}\right\rangle \int d y d z(y+z)\left(\frac{1}{z^{3}}+\frac{1}{y^{3}}\right)(1-y-z)\left(1+\frac{2 s}{T^{2}}\right) \delta\left(s-\bar{m}_{c}^{2}\right) \\
& -\frac{3 m_{c}\langle\bar{q} q\rangle}{256 \pi^{2}}\left\langle\frac{\alpha_{s} G G}{\pi}\right\rangle \int d y d z\left(\frac{y}{z^{2}}+\frac{z}{y^{2}}\right)(1-y-z)\left[1+\frac{2 s}{3} \delta\left(s-\bar{m}_{c}^{2}\right)\right] \\
& -\frac{m_{c}\langle\bar{q} q\rangle}{128 \pi^{2}}\left\langle\frac{\alpha_{s} G G}{\pi}\right\rangle \int d y d z\left[1+\frac{2 s}{3} \delta\left(s-\bar{m}_{c}^{2}\right)\right] \\
& -\frac{m_{c}\langle\bar{q} q\rangle}{512 \pi^{2}}\left\langle\frac{\alpha_{s} G G}{\pi}\right\rangle \int d y\left[1+\frac{2 s}{3} \delta\left(s-\tilde{m}_{c}^{2}\right)\right] \text {, } \\
& \rho_{8}^{Z}(s)=-\frac{m_{c}^{2}\langle\bar{q} q\rangle\left\langle\bar{q} g_{s} \sigma G q\right\rangle}{32 \pi^{2}} \int d y\left(1+\frac{s}{T^{2}}\right) \delta\left(s-\tilde{m}_{c}^{2}\right) \\
& +\frac{\langle\bar{q} q\rangle\left\langle\bar{q} g_{s} \sigma G q\right\rangle}{64 \pi^{2}} \int d y s \delta\left(s-\tilde{m}_{c}^{2}\right) \text {, } \\
& \rho_{10}^{Z}(s)=-\frac{\left\langle\bar{q} g_{s} \sigma G q\right\rangle^{2}}{256 \pi^{2} T^{4}}\left(1-\frac{m_{c}^{2}}{T^{2}}\right) \int d y s^{2} \delta\left(s-\tilde{m}_{c}^{2}\right) \\
& -\frac{m_{c}^{4}\langle\bar{q} q\rangle^{2}}{288 T^{4}}\left\langle\frac{\alpha_{s} G G}{\pi}\right\rangle \int d y\left[\frac{1}{y^{3}}+\frac{1}{(1-y)^{3}}\right] \delta\left(s-\tilde{m}_{c}^{2}\right) \\
& +\frac{m_{c}^{2}\langle\bar{q} q\rangle^{2}}{96 T^{2}}\left\langle\frac{\alpha_{s} G G}{\pi}\right\rangle \int d y\left[\frac{1}{y^{2}}+\frac{1}{(1-y)^{2}}\right] \delta\left(s-\tilde{m}_{c}^{2}\right) \\
& +\frac{\left\langle\bar{q} g_{s} \sigma G q\right\rangle^{2}}{18432 \pi^{2}} \int d y\left(1+\frac{2 s}{T^{2}}\right) \delta\left(s-\tilde{m}_{c}^{2}\right) \\
& +\frac{m_{c}^{2}\langle\bar{q} q\rangle^{2}}{288 T^{6}}\left\langle\frac{\alpha_{s} G G}{\pi}\right\rangle \int d y s^{2} \delta\left(s-\tilde{m}_{c}^{2}\right), \\
& \rho_{0}^{X}(s)=\frac{1}{2048 \pi^{6}} \int d y d z y z(1-y-z)^{2}\left(s-\bar{m}_{c}^{2}\right)^{3}\left(6 s-\bar{m}_{c}^{2}\right) \\
& +\frac{1}{8192 \pi^{6}} \int d y d z y z(1-y-z)^{3}\left(s-\bar{m}_{c}^{2}\right)^{2}\left(33 s^{2}-18 s \bar{m}_{c}^{2}+\bar{m}_{c}^{4}\right), \\
& \rho_{3}^{X}(s)=\frac{m_{s}\langle\bar{s} s\rangle}{128 \pi^{4}} \int d y d z y z\left(s-\bar{m}_{c}^{2}\right)\left(7 s-2 \bar{m}_{c}^{2}\right) \\
& +\frac{m_{s}\langle\bar{s} s\rangle}{256 \pi^{4}} \int d y d z y z(1-y-z)\left(35 s^{2}-30 s \bar{m}_{c}^{2}+3 \bar{m}_{c}^{4}\right) \\
& +\frac{9 m_{s} m_{c}^{2}\langle\bar{s} s\rangle}{64 \pi^{4}} \int d y d z\left(s-\bar{m}_{c}^{2}\right) \text {, }
\end{aligned}
$$




$$
\begin{aligned}
& \rho_{4}^{X}(s)=-\frac{m_{c}^{2}}{6144 \pi^{4}}\left\langle\frac{\alpha_{s} G G}{\pi}\right\rangle \int d y d z\left(\frac{z}{y^{2}}+\frac{y}{z^{2}}\right)(1-y-z)^{2}\left(9 s-4 \bar{m}_{c}^{2}\right) \\
& -\frac{m_{c}^{2}}{6144 \pi^{4}}\left\langle\frac{\alpha_{s} G G}{\pi}\right\rangle \int d y d z\left(\frac{z}{y^{2}}+\frac{y}{z^{2}}\right)(1-y-z)^{3}\left[5 s-\bar{m}_{c}^{2}+\frac{4 s^{2}}{3} \delta\left(s-\bar{m}_{c}^{2}\right)\right] \\
& -\frac{1}{3072 \pi^{4}}\left\langle\frac{\alpha_{s} G G}{\pi}\right\rangle \int d y d z(y+z)(1-y-z)\left(s-\bar{m}_{c}^{2}\right)\left(20 s-7 \bar{m}_{c}^{2}\right) \\
& +\frac{1}{12288 \pi^{4}}\left\langle\frac{\alpha_{s} G G}{\pi}\right\rangle \int d y d z(y+z)(1-y-z)^{2}\left(35 s^{2}-30 s \bar{m}_{c}^{2}+3 \bar{m}_{c}^{4}\right), \\
& \rho_{5}^{X}(s)=-\frac{m_{s}\left\langle\bar{s} g_{s} \sigma G s\right\rangle}{768 \pi^{4}} \int d y y(1-y)\left(9 s-4 \tilde{m}_{c}^{2}\right) \\
& -\frac{m_{s}\left\langle\bar{s} g_{s} \sigma G s\right\rangle}{256 \pi^{4}} \int d y d z y z\left[5 s-\bar{m}_{c}^{2}+\frac{4 s^{2}}{3} \delta\left(s-\bar{m}_{c}^{2}\right)\right]-\frac{9 m_{s} m_{c}^{2}\left\langle\bar{s} g_{s} \sigma G s\right\rangle}{256 \pi^{4}} \int d y, \\
& \rho_{6}^{X}(s)=-\frac{3 m_{c}^{2}\langle\bar{s} s\rangle^{2}}{32 \pi^{2}} \int d y \\
& \rho_{7}^{X}(s)=\frac{m_{s} m_{c}^{2}\langle\bar{s} s\rangle}{2304 \pi^{2}}\left\langle\frac{\alpha_{s} G G}{\pi}\right\rangle \int d y d z\left(\frac{z}{y^{2}}+\frac{y}{z^{2}}\right)\left(1-\frac{5 s}{T^{2}}\right) \delta\left(s-\bar{m}_{c}^{2}\right) \\
& +\frac{m_{s} m_{c}^{2}\langle\bar{s} s\rangle}{576 \pi^{2}}\left\langle\frac{\alpha_{s} G G}{\pi}\right\rangle \int d y d z\left(\frac{z}{y^{2}}+\frac{y}{z^{2}}\right)(1-y-z)\left(\frac{1}{4}+\frac{s}{T^{2}}-\frac{s^{2}}{T^{4}}\right) \delta\left(s-\bar{m}_{c}^{2}\right) \\
& +\frac{m_{s} m_{c}^{2}\langle\bar{s} s\rangle}{64 \pi^{2}}\left\langle\frac{\alpha_{s} G G}{\pi}\right\rangle \int d y d z \frac{1}{y^{2}}\left(2-y-\frac{y s}{T^{2}}\right) \delta\left(s-\bar{m}_{c}^{2}\right) \\
& -\frac{m_{s}\langle\bar{s} s\rangle}{4608 \pi^{2}}\left\langle\frac{\alpha_{s} G G}{\pi}\right\rangle \int d y\left[14+13 s \delta\left(s-\tilde{m}_{c}^{2}\right)\right] \\
& +\frac{m_{s}\langle\bar{s} s\rangle}{1152 \pi^{2}}\left\langle\frac{\alpha_{s} G G}{\pi}\right\rangle \int d y d z(y+z)\left[\frac{3}{4}+\left(s+\frac{s^{2}}{T^{2}}\right) \delta\left(s-\bar{m}_{c}^{2}\right)\right] \\
& +\frac{m_{s} m_{c}^{2}\langle\bar{s} s\rangle}{256 \pi^{2}}\left\langle\frac{\alpha_{s} G G}{\pi}\right\rangle \int d y\left(1+\frac{s}{T^{2}}\right) \delta\left(s-\tilde{m}_{c}^{2}\right), \\
& \rho_{8}^{X}(s)=\frac{3 m_{c}^{2}\langle\bar{s} s\rangle\left\langle\bar{s} g_{s} \sigma G s\right\rangle}{64 \pi^{2}} \int d y\left(1+\frac{s}{T^{2}}\right) \delta\left(s-\tilde{m}_{c}^{2}\right), \\
& \rho_{10}^{X}(s)=-\frac{3 m_{c}^{2}\left\langle\bar{s} g_{s} \sigma G s\right\rangle^{2}}{512 \pi^{2} T^{6}} \int d y s^{2} \delta\left(s-\tilde{m}_{c}^{2}\right) \\
& +\frac{m_{c}^{2}\langle\bar{s} s\rangle^{2}}{48 T^{2}}\left\langle\frac{\alpha_{s} G G}{\pi}\right\rangle \int d y \frac{1}{y^{2}}\left(1-\frac{y s}{2 T^{2}}\right) \delta\left(s-\tilde{m}_{c}^{2}\right) \\
& +\frac{\left\langle\bar{s} g_{s} \sigma G s\right\rangle^{2}}{3072 \pi^{2} T^{2}} \int d y s \delta\left(s-\tilde{m}_{c}^{2}\right) \\
& -\frac{m_{c}^{2}\langle\bar{s} s\rangle^{2}}{192 T^{6}}\left\langle\frac{\alpha_{s} G G}{\pi}\right\rangle \int d y s^{2} \delta\left(s-\tilde{m}_{c}^{2}\right),
\end{aligned}
$$

where $\int d y d z=\int_{y_{i}}^{y_{f}} d y \int_{z_{i}}^{1-y} d z, \int d y=\int_{y_{i}}^{y_{f}} d y, y_{f}=\frac{1+\sqrt{1-4 m_{c}^{2} / s}}{2}, y_{i}=\frac{1-\sqrt{1-4 m_{c}^{2} / s}}{2}, z_{i}=\frac{y m_{c}^{2}}{y s-m_{c}^{2}}, \bar{m}_{c}^{2}=\frac{(y+z) m_{c}^{2}}{y z}, \tilde{m}_{c}^{2}=\frac{m_{c}^{2}}{y(1-y)}$, $\int_{y_{i}}^{y_{f}} d y \rightarrow \int_{0}^{1} d y, \int_{z_{i}}^{1-y} d z \rightarrow \int_{0}^{1-y} d z$ when the $\delta$ functions $\delta\left(s-\bar{m}_{c}^{2}\right)$ and $\delta\left(s-\tilde{m}_{c}^{2}\right)$ appear. 
[1] S. K. Choi et al., Phys. Rev. Lett. 91, 262001 (2003).

[2] M. Tanabashi et al., Phys. Rev. D 98, 030001 (2018).

[3] H. X. Chen, W. Chen, X. Liu, and S. L. Zhu, Phys. Rep. 639, 1 (2016); R. F. Lebed, R. E. Mitchell, and E. S. Swanson, Prog. Part. Nucl. Phys. 93, 143 (2017); A. Esposito, A. Pilloni, and A. D. Polosa, Phys. Rep. 668, 1 (2017); F. K. Guo, C. Hanhart, U. G. Meissner, Q. Wang, Q. Zhao, and B. S. Zou, Rev. Mod. Phys. 90, 015004 (2018); A. Ali, J. S. Lange, and S. Stone, Prog. Part. Nucl. Phys. 97, 123 (2017); S. L. Olsen, T. Skwarnicki, and D. Zieminska, Rev. Mod. Phys. 90, 015003 (2018); Y. R. Liu, H. X. Chen, W. Chen, X. Liu, and S. L. Zhu, Prog. Part. Nucl. Phys. 107, 237 (2019); N. Brambilla, S. Eidelman, C. Hanhart, A. Nefediev, C. P. Shen, C. E. Thomas, A. Vairo, and C.Z. Yuan, arXiv:1907.07583.

[4] R. M. Albuquerque, J. M. Dias, K. P. Khemchandani, A. M. Torres, F. S. Navarra, M. Nielsen, and C. M. Zanetti, J. Phys. G 46, 093002 (2019).

[5] R. D. Matheus, S. Narison, M. Nielsen, and J. M. Richard, Phys. Rev. D 75, 014005 (2007).

[6] Z. G. Wang, Eur. Phys. J. C 63, 115 (2009); W. Chen and S. L. Zhu, Phys. Rev. D 83, 034010 (2011); J. R. Zhang, M. Zhong, and M. Q. Huang, Phys. Lett. B 704, 312 (2011); J. R. Zhang, Phys. Rev. D 87, 116004 (2013); C. F. Qiao and L. Tang, Eur. Phys. J. C 74, 2810 (2014); Z. G. Wang and Y. F. Tian, Int. J. Mod. Phys. A 30, 1550004 (2015); Z. G. Wang, Eur. Phys. J. C 78, 933 (2018); Z. G. Wang, Eur. Phys. J. C 79, 29 (2019).

[7] Z. G. Wang and T. Huang, Phys. Rev. D 89, 054019 (2014).

[8] Z. G. Wang, Eur. Phys. J. C 74, 2874 (2014).

[9] Z. G. Wang and T. Huang, Nucl. Phys. A930, 63 (2014); Z. G. Wang, Eur. Phys. J. C 79, 489 (2019).
[10] F. S. Navarra and M. Nielsen, Phys. Lett. B 639, 272 (2006); J. M. Dias, F. S. Navarra, M. Nielsen, and C. M. Zanetti, Phys. Rev. D 88, 016004 (2013); W. Chen, T. G. Steele, H. X. Chen, and S. L. Zhu, Eur. Phys. J. C 75, 358 (2015); S. S. Agaev, K. Azizi, and H. Sundu, Phys. Rev. D 93, 074002 (2016); Z. G. Wang and J. X. Zhang, Eur. Phys. J. C 78, 14 (2018); H. Sundu, S. S. Agaev, and K. Azizi, Eur. Phys. J. C 79, 215 (2019); Z. G. Wang, Int. J. Mod. Phys. A 34, 1950110 (2019).

[11] Z. G. Wang and T. Huang, Eur. Phys. J. C 74, 2891 (2014); Z. G. Wang, Eur. Phys. J. C 74, 2963 (2014).

[12] Z. G. Wang, Chin. Phys. C 41, 083103 (2017).

[13] W. Lucha, D. Melikhov, and H. Sazdjian, Phys. Rev. D 100, 014010 (2019); 100, 074029 (2019).

[14] H. J. Lee and N. I. Kochelev, Phys. Rev. D 78, 076005 (2008).

[15] L. D. Landau, Nucl. Phys. 13, 181 (1959).

[16] N. Gray, D. J. Broadhurst, W. Grafe, and K. Schilcher, Z. Phys. C 48, 673 (1990).

[17] F. K. Guo, L. Liu, U. G. Meissner, and P. Wang, Phys. Rev. D 88, 074506 (2013).

[18] F. K. Guo, X. H. Liu, and S. Sakai, arXiv:1912.07030.

[19] M. A. Shifman, A. I. Vainshtein, and V. I. Zakharov, Nucl. Phys. B147, 385 (1979); B147, 448 (1979).

[20] L. J. Reinders, H. Rubinstein, and S. Yazaki, Phys. Rep. 127, 1 (1985).

[21] P. Colangelo and A. Khodjamirian, in, QCD Sum Rules, a Modern Perspective, edited by M. Shifman, At the Frontier of Particle Physics Vol. 3 (World Scientific, Singapore, 2001), pp. 1495-1576, https://doi.org/10.1142/ 9789812810458_0033.

[22] S. Narison and R. Tarrach, Phys. Lett. 125B, 217 (1983).

[23] Z. G. Wang, Eur. Phys. J. C 75, 427 (2015).

[24] E. J. Eichten and C. Quigg, Phys. Rev. D 99, 054025 (2019). 